\title{
Sauf le nom : nommer les Digital Humanities
}

\section{$1 \quad$ Préambule}

Avant d'examiner l'écriture digitale elle-même au chapitre 3, le chapitre 2 va s'interroger sur le cadre général de l'avènement des digital humanities en enquêtant sur leur nom et en se demandant en particulier ce qui arrive à cette expression lorsqu'elle est traduite en français, dans sa double version « humanités numériques » et « humanités digitales ». Cette première partie de chapitre aboutira à scruter en profondeur le mot « humanité(s) », pour analyser le retour de l'usage de son pluriel désuet en français, par-delà le débat autour du binôme numérique/digital.

La deuxième partie de ce chapitre conduira à analyser un autre mot, un autre nom : l'esprit. En effet, c'est à ce terme ou concept que conduit la piste suggérée par Milad Doueihi et que j'ai annoncé vouloir suivre : revisiter la généalogie des digital humanities en partant de l'article d'Alan Turing. Ou, comme le formule exactement Doueihi : «Il est peut-être temps, sans diminuer l'importance de l'entreprise du père Busa, de proposer une autre généalogie aux humanités numériques. Car il se trouve qu'un des textes fondateurs de l'informatique elle-même soulève en grande partie les questions qui nous concernent aujourd'hui. Il suffit de relire « Computing Machinery and Intelligence » d'Alan Turing pour retrouver une partie importante des questionnements épistémologiques et philosophiques, voire même méthodologiques, qui sont toujours d'une grande actualité ${ }^{1}$. L'article de Turing va nous conduire à prêter attention à l'esprit, une notion qu'on trouve logée dans des traductions différentes de digital humanities.

L'hébreu désigne en effet les humanités digitales par l'expression Ruaḥ Digitalit, littéralement «l'esprit digital», bien qu'un site web indique ce nom comme «intraduisible $»^{2}$. Difficile en tous cas d'oublier que la Ruah est le

1 Doueihi, « Quête et enquête », p. 8-9; il est fait référence à ce passage au chapitre 1, p. 31, mais sans qu'il ne soit cité.

2 Voir I. Galina, « Geographical and linguistic diversity in the Digital Humanities », Literary and Linguistic Computing 29.3 (2014), p. 307-316. Au moment de la rédaction de ces pages, le site de l'Association israélienne en DH, Ruah Digitalit, ne fonctionne pas (http://thedigin.org; inaccessible en tous cas depuis le 20.01.18). Mais un site cartographiant les associations DH la présente ainsi : « Ruah Digitalit is the untranslatable name of an initiative for the promotion of digital humanities in Israel, with the vision to become a local node in the vast and growing 
terme hébreu désignant l'Esprit dans la Bible hébraïque (voir Gn 1,2). L'allemand, quant à lui, parle de digitale Geisteswissenschaften, ou parfois de l'« esprit digital », der digitale Geist, comme dans ce titre d'un article paru en 2016 dans le Tagesspiegel, « der digitale Geist erwacht »3 ${ }^{3}$ « l'esprit digital s'éveille », une expression qui peut rappeler l'héritage hégélien. L'allemand a, avec Geist, un terme équivalent à l'« esprit » en français, recouvrant tant spirit que mind, et présent de longue date dans le nom même des sciences humaines, die Geisteswissenschaften. La piste ouverte par l'article de Turing autour de l'esprit fait sens en regard de ces deux dénominations des DH dans d'autres langues. Il faut creuser.

Ce chapitre sera donc attentif aux mots et à la langue des digital humanities. Il portera son attention en particulier à ces deux noms, humanités et esprit. A l'heure où plusieurs chercheurs scrutent l'histoire de l'émergence des humanités numériques en francophonie ${ }^{4}$, la perspective choisie ici s'inscrit dans l'héritage de Jacques Derrida. Il avait magistralement souligné l'importance du nom dans Saufle nom:

Le nom : qu'appelle-t-on ainsi ? qu'entend-t-on sous le nom de nom ? Et qu'arrive-t-il quand on donne un nom ? Que donne-t-on alors? On n'offre pas une chose, on ne livre rien et pourtant quelque chose advient qui revient à donner, comme l'avait dit Plotin du Bien, ce qu'on n'a pas. Que se passe-t-il surtout quand il faut surnommer, re-nommant là où, justement, le nom vient à manquer? Qu'est-ce qui fait du nom propre une sorte de surnom, de pseudonyme ou de cryptonyme à la fois singulier et singulièrement intraduisible $?^{5}$

C'est bien de cet « intraduisible » dont il semble être question lorsque digital humanities tente de passer dans une autre langue, en français en particulier : « quelque chose advient qui revient à donner [...] ce qu'on n'a pas », lorsqu'on

network of the Digital Humanities » (Ruah Digitalit, <http://workshops.elotroalex.com/ beirut_a/neatline/fullscreen/dh-orgs\#records/52>).

3 Voir par exemple cet article du Tagesspiegel: A. Herbold, «Der digitale Geist erwacht », Der Tagesspiegel 15 Juin 2016, <http://www.tagesspiegel.de/wissen/digital-humanities-der-digi tale-geist-erwacht $/ 13738828 . h t m l>$.

4 Voir par exemple A. Berra, « Pour une histoire des humanités numériques » (2015); Le Deuff, Les humanités digitales, 2018. Ou encore une conférence donnée le 26 janvier 2018 à l'EHESS par Jérôme Valluy : « De l'historique des humanités numériques francophones (2007-2017) aux prémices d'une définition pluraliste des humanités numériques », <http://udpn.fr/spip.php? article272> ; P. Mounier, Les humanités numériques. Pour une histoire critique (2018).

5 J. Derrida, Sauf le nom, Galilée, 1993. Ce passage se trouve à la première page du feuillet Prière d'insérer qui se trouve glissé au début du volume. 
donne un nom, selon les termes de Derrida. Les deux parties de ce chapitre vont enquêter sur ce que n'aurait pas digital humanities, et ce dont ce nom se trouve gratifié en passant dans d'autres langues : ces « humanités » digitales ou numériques, en français, et cet « esprit » véhiculé par l'allemand et l'hébreu, mis en avant sous la plume de Turing. Cette approche via les noms et les mots est d'ordre généalogique et veut apporter une contribution complémentaire aux travaux de celles et ceux qui ont entamé la quête complexe d'une histoire des digital humanities, une tâche de longue haleine, décrite par Aurélien Berra comme forcément collective ${ }^{6}$.

\section{Première partie : Lost in translation? L'odyssée de digital humanities en français}

\subsection{Introduction ${ }^{7}$}

A première vue, le succès international de l'expression digital humanities semble avoir contribué à asseoir une fois de plus la domination linguistique de l'anglais sur les autres langues dans le milieu académique, en sciences humaines qui plus est. Discuté pour la première fois dans un article publié en $2004^{8}$ - mais déjà présent dans une conférence en ligne de $2002^{9}-$, analysé

6 Berra, «Pour une histoire des humanités numériques», p. 621 : écrire l'histoire des humanités numériques est une tâche « à la fois scientifique, culturelle, institutionnelle et technologique. Comment produire collectivement une telle histoire ? En profitant de l'ouverture d'un dialogue entre spécialités et en rassemblant les matériaux hérités sous bénéfice d'inventaire, pour commencer». Berra renvoie notamment à McCarty et Turner pour l'état de la recherche : W. McCarty, Humanities Computing, Palgrave Macmillan, 2005; F. Turner, Aux sources de l'utopie numérique. De la contre-culture à la cyberculture, L. Vannini (trad.), C\&F éditions, 2012. Pierre Mounier vient d'écrire une histoire des humanités numériques que je n'ai pas pu consulter avant la rédaction du présent ouvrage (P. Mounier, Les humanités numériques. Pour une histoire critique, EMSH éditions, octobre 2018).

7 Cette première partie de chapitre traduit plusieurs passages de cet article publié en anglais, avec l'aimable autorisation de l'éditeur : Clivaz, « Lost in Translation? » (2017).

8 Kirschenbaum, M. G., "What Is Digital Humanities and What's It Doing in English Departments? », ADE Bulletin 150 (2010), p. 55-61, ici p. 56 ; <http://mkirschenbaum.files. wordpress.com/2011/o3/ade-final.pdf $>$.

9 Merci à Amos Bairoch (Bioinformatique, Université de Genève) de m'avoir transmis cette référence : I. Johnson, « Mapping the Humanities: The Whole is Greater Than The Sum Of Its Parts », final 2002 draft presented at Computing Arts 2001:Digital Resources for Research in the Humanities, Sydney: University of Sydney ; la conférence de Johnson est annoncée publiée dans sa bibliographie personnelle, mais les pages ne sont pas indiquées. Il s'avère impossible de trouver une trace de la publication en question (I. Johnson, « Mapping the Humanities: The Whole is Greater Than The Sum Of Its Parts », Computing Arts 2001: 
sans fin dans les colloques et conférences du domaine, l'appellation digital humanities, ou DH, s'est retrouvée avec une vitesse fulgurante associée à des titres de postes académiques, de degrés académiques, ou pour qualifier des centres, des laboratoires et des projets de recherche ${ }^{10}$. Si les autres langues ont été conduites à accueillir cette expression, ses traductions gagneraient à être étudiées de manière systématique, car on pourrait sans doute y observer de formidables effets retour de signification et de refonte épistémologique. Autrement dit, en passant d'une langue à l'autre, digital humanities peut muter au point de signifier quelque chose de partiellement différent, ou d'amener à d'autres potentialités de sens.

Nous souhaitons démontrer ici que le retour de l'usage du terme d'« humanités » en français, devenu démodé, est l'élément le plus significatif de la double expression francophone « humanités numériques » ou « humanités digitales », qui cherche à rendre digital humanities. Pour en prendre la mesure, l'introduction de cette première partie esquissera les contours généraux de la problématique de l'expression digital humanities longuement discutée sous la plume des collègues anglo-saxons. Le deuxième point résumera ensuite les principaux arguments des débats qui confrontent humanités digitales et humanités numériques en francophonie. Le troisième point se penchera sur l'arrière-plan étymologique du terme désuet humanités, qui indique la thématique du corps comme centrale dans la traduction française de l'expression digital humanities.

Le foisonnement des débats qui ont cours autour de la définition et de la délimitation de l'expression digital humanities est bien connu de la recherche. Toutefois, une étape importante a été franchie dans la perception de cette notion au cours des dernières années. En 2009, Patrick Svensson a proposé de discerner un «tournant discursif des humanities computing aux digital humanities $»^{11}$. Il considérait alors que les tensions entre ces deux expressions étaient nées d'un manque d'engagement des humanities computing avec le « 'digital' en tant qu'objet d'étude » ${ }^{12}$. Dans ces références à Svensson, je choisis de conserver ces deux expressions en anglais, pour éviter de mettre trop rapidement en équivalence humanities computing et «humanités numériques», ainsi qu'« humanités digitales» avec digital humanities. En effet, en français

Digital Resources for Research in the Humanities, Sydney: University of Sydney, 2003 ; mentionnée ici : <https://sydney.edu.au/arts/staff/publications/ian.johnson.pdf>).

11 Svensson, P., «Humanities Computing as Digital Humanities », DHQ 3 (2009/3), § 1-62, <http://www.digitalhumanities.org/dhq/vol/3/3/000065/000065.html>; abstract. 
le donné est plus complexe que cette mise en équation linguistique, nous le verrons.

Sept ans plus tard, dans le deuxième volume de la série Digital Debates, Steven Jones, s'engageant dans le débat, utilisera le terme d'everted (litt. «évasé » ou « retourné vers l'extérieur ») pour qualifier l'extension, voir l'omniprésence de ce « digital», considéré par Svensson comme négligé dans le nom humanities computing:

Les nouvelles pratiques et champs d'intérêts dans les sciences humaines pour l'informatique correspondent aux changements associés à l'extension (eversion) du cyberespace dans la culture au sens large. En un sens, les nouvelles digital humanities sont les humanities computing, évasées (everted). [...] Ce terme reflète aussi un plus grand changement : il implique une séparation entre les matériaux des sciences humaines manuscrits, livres, documents, cartes, œuvres d'art de toutes sortes et autres artefacts culturels - et l'informatique, pour [aller vers] une réalité mixte, caractérisée par des interactions dans les deux sens entre les deux domaines, les artefacts physiques et les médias digitaux ${ }^{13}$.

De manière générale, Jones présente cette «extension digitale » comme un phénomène positif, évolutif : l'avènement de l'expression digital humanities signale pour lui une compréhension plus en profondeur de ce phénomène d'eversion, d'extension du digital dans la culture générale ${ }^{14}$. Cette extension est présentée en revanche avec la plus vive inquiétude par Bernard Stiegler à travers le terme de "disruption », qu'il commente souvent et qui figure dans le titre de son ouvrage de 2016, Dans la disruption. Comment ne pas devenir fou?

13 S. E. Jones, « The Emergence of the Digital Humanities (the Network Is Everting) », dans Debates in the Digital Humanities, M. K. Gold - L. Klein (éd.), University Minesota Press, 2016, <http://dhdebates.gc.cuny.edu/debates/text/52> : «New practices and areas of interest for computing in the humanities correspond to changes associated with the eversion of cyberspace in the culture at large. In one sense, the new digital humanities is humanities computing, everted. [...] The term also reflected a larger change : from implying a separation between the stuff of the humanities - manuscripts, books, documents, maps, works of art of all kinds, other cultural artefacts - and computing, to more of a mixed reality, characterized by two-way interactions between the two realms, physical artefacts and digital media ».

Je renonce ici à traduire eversion par « éversion », qui existe en français, mais est un terme médical avec un sens trop spécifique pour l'usage visé par Svensson ; Eversion, <http:// www.linternaute.com/dictionnaire/fr/definition/eversion/ $>$ : « retournement d'un tissu mou du corps humain vers l'extérieur». Le terme a aussi un sens ancien qui ne correspond pas à l'emploi d'eversion fait par Svensson : « destruction d'une ville, d'un état ou d'une pensée». 
Stiegler désigne par ce terme « l'accélération du phénomène de l'innovation », imposée, et qui est une «nouvelle forme de barbarie », un « facteur planétaire de désintégration sociale. Ce pouvoir automatique de désintégration réticulaire s'étend sur toute la Terre à travers ce qu'on appelle depuis quelques années la disruption $»^{15}$.

Il est à relever que Stiegler ne va pas donner d'étymologie ou d'historique à ce terme, présent pourtant depuis la fin du $18^{\mathrm{e}}$ siècle dans le Dictionnaire critique de la langue française (1787-1788) avec le sens de «rupture », et « utile aussi dans la Physique et dans l'Histoire naturelle », comme on l'apprend par l'outil de recherche en ligne Dictionnaire Vivant de la Langue Française ${ }^{16}$. Omettant ce détour via l'histoire des mots, dont Stiegler est pourtant féru dans le reste de l'ouvrage, il prend « disruption » dans toute la violence de son sens immédiat, «depuis quelques années », et lui donne une extension maximale - tout comme c'est le cas chez Jones pour eversion: «absolument tous les domaines sont concernés : toutes les pratiques, toutes les formes de vie, toutes les entreprises individuelles et collectives $»^{17}$. Je reviendrai au chapitre 3 sur l'analyse de cette disruption et la manière dont Stiegler y réagit ${ }^{18}:$ je prends note pour l'instant de cette extension maximale, similaire à celle contenue dans eversion. Elle le conduit à réclamer en conséquence un champ d'études également extensif à tout domaine, les digital studies, les études digitales qui devraient avoir pour but de produire de «nouveaux programmes institutionnels aussi bien qu'industriels ${ }^{19}$.

C'est à ce point exact d'extension qu'il convient de scruter le nom des digital humanities. Sont-elles absorbables par les digital studies, dans cette extension du digital qui balaie à force de disruption tout ce qui gravite peu ou prou non seulement dans la culture mais aussi dans le monde environnemental, scientifique, économique ? Invités à prêter attention aux noms, à ce qui s'y joue et s'y dit, par l'opuscule magistral de Jacques Derrida Sauf le nom ${ }^{20}$, nous allons voir qu'au cœur des digital humanities, les sciences humaines ou humanités résistent, en s'attachant à observer le devenir de ce nom en français.

\footnotetext{
15 B. Stiegler, Dans la disruption. Comment ne pas devenir fou?, Les liens qui libèrent, 2016, p. 3,15 et 22 .

16 Dictionnaire Vivant de la Langue Française (DVLF), « Disruption », Université de Chicago, $<$ https://dvlf.uchicago.edu/mot/disruption>.

17 B. Stiegler, «Dans la disruption. La main, ses doigts, ce qu'ils fabriquent et au-delà », Etudes Digitales 1 (2016), p. 215-227 ; ici p. 221.

18 Voir chapitre 3, p. 99-100.

19 Stiegler, «Dans la disruption », p. 218.

20 Derrida, Sauf le nom.
} 
Le premier texte en français qui peut être perçu comme rassemblant les opinions d'une partie non négligeable des chercheurs de cette langue est le Manifeste des digital humanities, écrit à Paris en mai 2010 et qui avait fait le choix de garder ce nom en anglais ${ }^{21}$. Depuis, l'habitude de garder ce nom en anglais revient souvent sous la plume d'auteurs francophones, et j'en fais moi-même usage dans cet ouvrage. La pratique évolue parfois, comme chez Olivier Le Deuff qui rend compte du moment où il est passé de l'usage, en français, de digital humanities à celui d'« humanités digitales $»^{22}$. Le fait de différer la traduction de digital humanities peut se retrouver y compris dans un titre institutionnel, comme c'est le cas pour le centre autrichien en humanités numériques nommé Austrian Center for Digital Humanities, alors qu'il est implanté en pays germanophone. Le point est d'autant plus frappant que tous les autres instituts de l'Académie autrichienne des sciences, pour ce qui concerne les sciences humaines et sociales, portent un nom allemand (Geistes-, Sozial- und Kulturwissenschaften $)^{23}$.

Cet exemple renforce l'impression qu'il y a quelque chose dans le nom des DH qui n'est pas intégrable tel quel dans les sciences humaines traditionnelles, et qu'il peut effectivement advenir un effet de sens particulier au moment de sa traduction. De ce point de vue, «humanités numériques » n'a donc jamais suffi à rendre compte de ce qu'il y avait dans digital humanities, pointant sur le rapport cérébral aux chiffres $\mathrm{du}^{24}$ code. On y retrouve le manque d'engagement avec le digital comme objet d'étude souligné par Svensson dans l'expression humanities computing ${ }^{25}$. L'attachement exclusif de certains chercheurs à « humanités numériques » pour motif linguistique fait tout simplement l'impasse sur le binôme anglophone humanities computing et digital humanities. Elargir la réflexion à une approche multilingue permet d'avancer dans la perception de ce qui est à l'œuvre sous les mots.

Une expérience linguistique intéressante avait été conduite lors de la conférence $\mathrm{DH}_{2014}$ : l'appel à contributions, rédigé en anglais, avait eu pour particularité d'être traduit en vingt-trois langues, et plusieurs de ces langues avaient dû choisir des mots pour rendre compte du vocabulaire utilisé dans les DH

21 M. Dacos, « Manifeste des Digital humanities. ThatCamp Paris $2010 »$, mai 2010, <http:// tcp.hypotheses.org/318>.

22 O. Le Deuff, « Humanités digitales versus humanités numériques, les raisons d'un choix », Etudes Digitales 1 (2016), p. 263-264; ici p. 263.

23 Oesterresische Akademie der Wissenschaften (öAw), <https://www.oeaw.ac.at/acdh/acdhhome/>.

24 On verra plus loin que Cormerais et Gilbert opposent charnalité et calcul pour dire la différence entre humanités digitales et humanités numériques (F. Cormerais - J. A. Gilbert, «Introduction », Etudes Digitales 1 (2016), p. 11-16 ; ici p. 14 ; cité p. 55 ci-dessous).

Svensson, « Humanities Computing », §2. 
anglo-saxonnes ${ }^{26}$. Ce fut notamment le cas en arabe : David Wrisley et son équipe au Liban durent travailler à la traduction avec un soin particulier, choisissant des équivalences linguistiques pour donner un lieu de vie arabe aux expressions et concepts anglo-saxons ${ }^{27}$. Une telle expérience, unique dans l'histoire des colloques internationaux en Digital Humanities, a démontré à la fois la contrainte imposée par l'anglais aux autres langues, mais aussi le réel potentiel de créativité offert aux autres langues dans un tel exercice. C'est en tout cas ce que souligne l'exemple du français, qui reprend à son compte et transforme l'expression digital humanities, comme nous allons le voir. On devrait encourager des enquêtes similaires dans d'autres langues.

\subsection{L'odyssée francophone d'humanités digitales versus humanités numériques}

L'expression « humanités digitales » est une traduction française mot à mot de digital humanities, dont le premier usage apparaît à Bordeaux en 2008, par Valérie Carayol et ses collègues ${ }^{28}$. En parallèle et sans qu'il n'y ait eu d'échange entre les personnes concernées, une autre équipe de chercheurs a commencé à parler d'humanités digitales sur le campus de Lausanne dès la fin $2009^{29}$; j'ai conservé pour ma part cet usage. Quelques années plus tard, Valérie Carayol et Franc Morandi publièrent un ouvrage collectif qui devait porter le titre d'Humanités digitales, le tournant des sciences humaines ${ }^{30}$, mais qui au final se retrouva intitulé Le tournant numérique des sciences humaines et sociales ${ }^{31}$. Toutefois, l'introduction de l'ouvrage ne parle que d'humanités digitales, sans aucune mention ou explication de l'écart qui se trouve entre le titre du collectif et cette appellation reprise dans l'introduction ${ }^{32}$.

De tels changements, hésitations ou adaptations entre les deux traductions françaises de digital humanities sont perceptibles dans les circonstances les

26 DH2014, Multilingual Call for Papers, <https://dh2014.org/2013/08/31/call-for-proposalsonline/>.

27 Ces indications proviennent d'une discussion que j'ai eue avec David Wrisley.

28 Le Deuff, « Humanités digitales », p. 263.

29 Voir C. Clivaz, «Re: [Humanist] 24.697 published definitions », Humanist Discussion Group 24/700 (2011), <http://lists.digitalhumanities.org/pipermail/humanist/2011-Febru ary $/ 008743 \cdot h t m l>$.

30 Collectif, «Humanités numériques: une question de lexique », ThatCamp St-Malo 2013. Non actes de la non conférence, La Non-Collection, Editions de la maison de sciences de l'homme, 2014, §1-21, ici §1; <http://books.openedition.org/editionsmsh/2189>.

31 V. Carayol - F. Morandi (dir.), Le tournant numérique des sciences humaines et sociales, Maison des sciences de l'Homme d'Aquitaine, 2015.

32 Carayol - Morandi (dir.), Le tournant numérique, p. 9-12. 
plus diverses, notamment dans les titres académiques. En 2015, l'Université de Lausanne $(\mathrm{CH})$ a fait démarrer une spécialisation de master en « Humanités digitales $»^{33}$, pour la remplacer en 2016 par un nouveau master complet et interfacultaire en «Humanités numériques $»^{34}$. L'expression «humanités digitales » a souvent été critiquée, voire a pu faire l'objet du dédain d'un certain parisianisme, par exemple sous la plume de Milad Doueihi signalant en 2014 qu'il en était fait usage « dans certaines régions de la sphère francophone $»^{35}$. En 2016, Dominique Vinck estimait quant à lui que « l'expression humanités digitales risquait d'être abandonnée au détriment du projet intellectuel associé à son utilisation ${ }^{36}$.

D'opinion inverse, mais en 2016 aussi, Olivier Le Deuff a publié un billet de blog intitulé «Dix raisons de préférer digital à numérique » ${ }^{37}$, tandis que paraissait le premier numéro de la revue Etudes digitales, consacrant plusieurs pages à ce débat linguistique intra-francophone : la majorité des chercheurs y soutiennent le choix d'humanités digitales ${ }^{38}$. En 2018 , il publie également une monographie intitulée Les humanités digitales. Historique et développement. Il y justifie l'usage d'« humanités digitales » par l'ampleur de l'expression ${ }^{39}$, et le lien sémantique au « doigt ». La thèse de son ouvrage est en effet de proposer d'ancrer l'émergence des humanités digitales dans l'usage de l'index : «Selon notre approche, les humanités digitales débutent avec l'index. [...] Faire débuter les humanités digitales par l'index permet de rappeler que 'digital' se

33 Spécialisation de maîtrise en Humanités digitales, Université de Lausanne, <https://www. unil.ch/lettres/fr/home/menuinst/master-et-specialisation/ma-avec-specialisation/hu manites-digitales.html>; cette page était encore accessible au 14 mai 2017, mais ne l'était plus au 10 janvier 2018.

34 Maîtrise universitaire en humanités numériques, Université de Lausanne $(\mathrm{CH}),<\mathrm{https} / / /$ www.unil.ch/ssp/home/menuinst/formations/master/humanites-numeriques.html>.

35 Doueihi, «Préface. Quête et enquête », p. 8.

36 D. Vinck, Humanités numériques. La culture face aux nouvelles technologies, Le Cavalier Bleu, 2016, p. 9-10.

37 O. Le Deuff, «10 raisons de préférer digital à numérique », Le guide des égarés, 2016 ; $<$ http://www.guidedesegares.info/2016/01/31/10-raisons-de-preferer-digital-a-numerique/>.

$38 \quad$ F. Cormerais - J. A. Gilbert (dir.), Etudes Digitales 1 (2016), p. 13-15, 215-227 et 251-268.

39 Le Deuff, Les humanités digitales, p. 10 : « La question des humanités digitales dépasse [...] la seule vision informatique adossée aux sciences humaines et sociales. Réduire cette histoire à l'apparition des outils informatiques et à leur utilisation par les chercheurs en sciences humaines et sociales ne permet pas de comprendre les filiations ainsi que les nombreuses tentatives interdisciplinaires et transdisciplinaires qui ont permis d'introduire des méthodes de compréhension et d'analyse nouvelles ». 
rapporte initialement aux doigts. Ici, il s'agit de montrer que l'index est justement le doigt le plus important dans ce cadre ${ }^{40}$.

En amont de ces écrits de Le Deuff, le ThatCamp 2013 de St-Malo (FR) a représenté un moment-clé de la discussion francophone autour de l'expression digital humanities, lors de la pré-assemblée générale de la future association francophone des DH. A cette occasion, un séminaire avait été le cadre d'une vigoureuse discussion sur le nom de la future association, et sur les différences entre numérique et digital. Les actes, publiés très rapidement après, en ont gardé la trace sous la forme d'une écriture collective. Plusieurs arguments, de teneurs diverses, furent mis en avant: par exemple, Charlotte Touati rappelle que l'allemand, au contraire du français, est toujours prêt à accueillir de nouveaux mots anglais en son sein ; quant à Aurélien Berra, il a rappelé lucidement que l'attribution des budgets était souvent dépendante des appellations ${ }^{41}$. Le texte montre qu'un accord général s'est peu à peu dégagé en faveur d'un nom tierce pour l'association, qui ne soit ni humanités numériques, ni humanités digitales. Marin Dacos proposa Humanistica ${ }^{42}$, qui fut confirmé plus tard comme nom pour la toute jeune association ${ }^{43}$.

Une histoire aussi récente et intense signale-t-elle que cette question linguistique est plutôt à voir comme une « coquetterie française », ou montre-telle des enjeux sous-jacents d'importance? Les éléments qui sont mis en évidence dans les pages qui suivent montrent qu'on est loin d'un phénomène anecdotique, et qu'il faut souhaiter que les chercheurs continuent à explorer ce fait langagier en tant que manifestation culturelle de profonds déplacements dans notre rapport au savoir, à l'humain, au corps. Car la surprise qui est au rendez-vous de l'analyse est la présence du corps, mais pas seulement où on l'attendrait spontanément, soit dans le terme de « digital », qui porte l'écho du latin digitus, le doigt.

Le sens corporel de «digital » fait l'objet d'un large consensus parmi les chercheurs enclins à parler d'humanités digitales ou d'études digitales ${ }^{44}$. Pour Bernard Stiegler, « digital » signale dans cette expression « le rôle de la main, de ses doigts et de ce qu'ils fabriquent dans la formation de la pensée à travers les savoirs vivre, faire et concevoir ${ }^{45}$. Cette attention répétée à « digital » et à son

\footnotetext{
$40 \quad$ Le Deuff, Les humanités digitales, p. 35.

41 Collectif, « Humanités numériques », §17.

42 Collectif, « Humanités numériques », §19.

43 Humanistica, <http://www.humanisti.ca>.

44 Voir notamment Cormerais - Gilbert, Etudes digitales, p. 13-15, 215-227 et 251-268; Le Deuff, « 10 raisons »; Collectif, « Humanités numériques », §1-2 ; Clivaz, «Common era », p. 52-53.

Stiegler, «Dans la disruption », p. 217.
} 
sens corporel tranche avec le fait que fort peu de chercheurs se sont penchés sur le retour en français de ce mot désuet et démodé, les humanités, présent pourtant dans les deux expressions en question ${ }^{46}$.

En 2012, Aurélien Berra, rappelant les racines de l'expression « faire ses humanités » dans l'humanisme de la Renaissance, a suggéré qu'« en passant de humanities à 'humanités', on ne désigne évidemment pas la même chose. Les découpages disciplinaires sont différents. Selon les catégories du CNRS, les humanities correspondraient aux sciences humaines et sociales, c'est-à-dire à un ensemble assez large qui ne se superpose pas avec ce qui existe dans d'autres traditions culturelles, universitaires et scolaires. On s'éloigne ainsi de la référence à des disciplines établies, pour nommer un phénomène complexe ${ }^{47}$. Je partage absolument cette analyse de la complexification du phénomène, mais serais plus réservée quant à l'idée de lire d'emblée les sciences sociales comme incluses lors du passage de «sciences humaines » à « humanités », quand bien même Berra s'appuie ici sur les « catégories du CNRS », non référencées dans l'article, toutefois.

Il est à relever par exemple que les sciences sociales ne font pas partie de la définition de l'organisation faîtière internationale en digital humanities, $\mathrm{ADHO}^{48}$, ni de ses organisations constitutives, telles EADH, l'organisation européenne ${ }^{49}$. En ce qui concerne les infrastructures de recherche européenne, les ERIC (European Research Infrastructure Consortium) ${ }^{50}$, les sciences sociales sont principalement représentées par $\operatorname{CESSDA}^{51}$, tandis que les sciences humaines, présentes à ce niveau de recherche seulement sous leur modalité digitalisée, sont représentées par DARIAH et CLARIN ${ }^{52}$. Si telle est la répartition des domaines au sein de ces institutions, du côté des chercheurs l'aspiration à étendre les frontières grandit. Cormerais et Gilbert vont dans le même sens

46 Voir essentiellement A. Berra, «Faire des Humanités Numériques», dans Read/Write Book 2, P. Mounier (éd.), OpenEdition Press, 2012, p. 25-43 ou §1-56 pour l'édition numérique ; ici § 2-3; <http://books.openedition.org/oep/238> ; C. Clivaz - D. Vinck, «Introduction. Des humanités délivrées pour une littérature plurielle », Les Cahiers du numérique 10/3 (2014), p. 9-16 ; ici p. 9 ; Clivaz, « De 'numérique' à 'digital' », p. 255.

47 Berra, «Faire des Humanités Numériques », §3.

48 ADHO (Alliance of Digital Humanities Organizations), <https: //www.adho.org >.

49 EADH (European Association for Digital Humanities), <https://eadh.org/>.

50 ERIC (European Research Infrastructure Consortium), <https://ec.europa.eu/research/in frastructures/index_en.cfm?pg=home>.

51 CESSDA (Consortium of European Social Science Data Archives), <https://cessda.net/>.

$5^{2}$ DARIAH (Digital Research Infrastructure for Arts and Humanities), <https://www.dariah. eu/>; ClARIN (Common Language Resources and Technoloca Infrastructure), <https:// www.clarin.eu/>. 
que Berra, précisément en raison de la dimension charnelle qu'ils entendent dans le terme « digital» :

Le choix de «digital» tente de restituer la sensation du toucher, ce qui produit une double digitalisation : celle du nombre mais aussi celle du contact avec cette matière calculée. Le terme « digital » réinstalle la dimension phénoménologique qui fait que toute pensée se trouve à un moment incarnée. Notre relation au calcul, à ce qu'on désigne parfois comme l'immatériel malgré la matérialité bien tangible des outils, se présente toujours, d'une manière ou d'une autre, dans une charnalité dont la limite se tient dans la plénitude incurvée du bout des doigts. Cette ambivalence de la charnalité et du calcul, nous la revendiquons comme l'expression la plus adéquate de la tension qui traverse la totalité des études digitales. Celles-ci ne sont en effet réductibles ni au code, ni aux disciplines qui constituent le domaine des sciences humaines ${ }^{53}$.

Cormerais et Gilbert avancent ici le terme fort de charnalité, et comme Olender mettent en scène la tension immatériel-matériel ${ }^{54}$. Il en va globalement d'une extension de tout ce qui est touché par le « digital », similaire à l'usage d'eversion (Jones) ou de disruption (Stiegler). Ne faut-il donc pas prendre acte de cette extension en cours et conclure que le retour du terme « humanités » nous conduit inexorablement aux études digitales prêtes à accueillir les derniers des mohicans des humanistes? L'usage de « digital » vient-il enfin ressouder la sphère corporelle à ces sciences humaines de papier devenues trop cérébrales, immergées dans leurs livres, en exil loin de la sphère sociale et anthropologique?

C'est en premier lieu mon expérience de travail interdisciplinaire, en tant que cheffe de projets en humanités digitales au sein d'un institut de bioinformatique, qui me fait clairement dire que les sciences humaines, et ce qu'elles deviennent sous le label d'humanités digitales, ont leur résistance propre et leur questionnement particulier. Ce sentiment, né de la pragmatique quotidienne de la recherche, se trouve conforté par ce qu'on découvre en s'immergeant dans le vocable même des «humanités », ce terme encore désuet il y a peu.

\subsection{Le terme « humanités » et ses significations oubliées}

Le pluriel d'«humanités » a été presque oublié en français dans les dernières décennies jusqu'à son récent succès dans le monde des humanités numériques.

53 Cormerais - Gilbert, «Introduction », p. 13-14.

54 Voir Olender, Un fantôme, p. 28 ; commenté au chapitre 1, p. 24-28. 
Le pluriel devient d'usage courant au $17^{\mathrm{e}}$ siècle pour désigner un parcours de formation, tel que défini par exemple dans l'édition de 1831 du Dictionnaire de l'Académique française : «On appelle HUMANITES, ce qu'on apprend ordinairement dans les Collèges jusqu'à la Philosophie exclusivement. Il a fait ses humanités. Il a achevé ses humanités. Enseigner les humanités »55. A noter que la philosophie n'est pas incluse : sont concernées essentiellement les langues classiques et la grammaire. Toutefois, le pluriel d'humanités était déjà présent dans l'histoire de ce mot avant l'âge classique et son lien à l'éducation.

Le Dictionnaire de l'ancienne langue française et de tous ses dialectes du IX $a u X V^{e}$ siècle, dans son volume complémentaire, indique au pluriel les significations suivantes : «biens profanes, choses du monde » («de temptables humanitez »), et au singulier la «nature humaine », le corps, par opposition à l'âme et à la déité56. Les sens de base du terme « humanité » dans ces siècles sont répertoriés au volume 4 de ce dictionnaire, et sont référencés comme «la vie » et les «parties honteuses » ou sexuelles (Adam qui cache son humanité), ou encore la prévenance, le bon accueil («faire moult de humanitez») ${ }^{57}$. Cette pluralité de sens, corporel inclus, se retrouve déclinée ensuite dans Le dictionnaire de référence pour la langue de la Renaissance, du $15^{e}$ au $17^{e}$ siècle. On y lit à nouveau les sens d'« urbanité, bienveillance, affabilité » et de «plaisir délicat », et aussi celui de «parties sexuelles » et de « corps de l'homme», avec cet usage pluriel dans cet exemple tiré de Rabelais ${ }^{58}$ : « Pourquoy plus toust ne transportons nous nos humanitez en belle cuisine de Dieu ? ${ }^{59}$.

55 Société des grammairiens, « Humanité », Dictionnaire de l'Académie française $A-K$, vol. 1 , Paris : Guillaume, 1831, p. 705; <https://tinyurl.com/yddskst4>.

$56 \quad$ F. Godefroy, « Humanité », dans Dictionnaire de l'ancienne langue française et de tous ses dialectes du IX $X^{e}$ au $X V^{e}$ siècle, F. Godefroy (éd.), vol. IV, Paris : F. Vieweg, 1885 \& Slatkine, 1982, p. 526 ; vol. IX, Paris : Emile Bouillon, 1898 \& Slatkine, 1982, p. 774 ; <http://gallica. bnf.fr/ark:/12148/bpt6k50634Z>. Ici, vol. IX, p. 774 ; <http://gallica.bnf.fr/ark:/12148/bpt $6 \mathrm{k} 41952 / \mathrm{f}_{77} 8$.image $>$.

Godefroy, « Humanité », vol. IV, p. $5^{26} ;<$ http://gallica.bnfffr/ark:/12148/bpt6k419oh/f530. image $>$.

58 Rabelais, Le Quart livre des faitcs et dicts hérö̈ques du bon Pantagruel X (1552), dans CEuvres complètes, tome 2, Ch. Marty-Laveaux (éd.), Paris : A. Lemerre, 1870, p. 306 : « Vertu Dieu, da jurandi, pourquoy plus toust ne transportons nous nos humanitez en belle cuisine de Dieu ? Et là ne confyderons le branlement des broches, l'harmonie des contrehaftiers, la pofition des lardons, la temperature des potaiges, les preparatifz, du deffert, l'ordre du feruive du vin? Beati immaculate in via. C'eft matiere de breuaire». Il est à noter qu'Huguet supprime les deux jurons initiaux dans la phrase citée.

59 E. Huguet, « Humanité », dans Le dictionnaire de référence pour la langue de la Renaissance, du $15^{e}$ au $17^{e}$ siècle, C. Blum (éd.), 1925-1967, Classiques Garnier Numérique, Editions Champion Électronique, pas d'année; <https://classiques-garnier.com/huguet-diction naire-du-xvie-s.html>. 
Ce dictionnaire, sous la plume d'Huguet, décline de manière impressionnante les significations corporelles d'humanité(s). Par exemple, le «moule d'humanité » désigne le « ventre de la femme », sens qu'Huguet complète de cet exemple: «ne desirant ressembler les femmes de ses anciens Perses qui presenterent leurs moulles d'humanité a leurs enfants et parents qui fuyoient de la bataille. Prem. acte du Synode noct. $15^{{ }^{60}}$. Etape supplémentaire : ce moule de chair, par extension, se met aussi à désigner les caractères d'imprimerie, et «mettre en moule » se mettra à signifier «imprimer, faire imprimer ${ }^{61}$. Un lien est tissé ici entre le corps et les lettres, entre «moule d'humanité » et « moule d'imprimerie ».

Aussi surprenant que ce soit, alors que bon nombre de chercheurs accentuent l'aspect corporel, voire charnel, du terme digital dans « humanités digitales », à ma connaissance ${ }^{62}$, ce potentiel de sens incarné contenu dans l'histoire étymologique du terme humanités, avant l'âge classique, ne semble pas avoir été relevé. On a vu ci-dessus qu'Olivier Le Deuff avait choisi de donner comme point de départ d'un historique des humanités digitales l'invention de l'index, en lien au sens de «doigt », évoqué par l'adjectif « digital » ${ }^{63}$. C'est également « digital » que Gilbert et Cormerais lient à charnalité64, mais ce lien semble tout aussi profondément incrusté dans l'histoire étymologique d'«humanité(s)».

Il est fascinant de constater que des significations peuvent être associées à des mots, puis oubliées, ou en grande partie oubliées : les attaches entre les mots et les choses, selon le binôme de Foucault, peuvent varier, parfois considérablement. Le lien ancestral d'humanité(s) à la chair persiste encore en de rares usages contemporains, par exemple sous la plume de la psychiatre MarieRose Moro. En 2008, elle a écrit un article portant sur les corps féminins intitulé «L'infinie matière des humanités. Corps, espace, temps, au féminin pluriel ${ }^{65}$. Humanités est ici référé comme le corps-matière, celui des femmes

$60 \quad$ Huguet, «Humanité », Le dictionnaire de référence, <https://classiques-garnier.com/hu guet-dictionnaire-du-xvie-s.html>.

61 Huguet, «Humanité », Le dictionnaire de référence, <https://classiques-garnier.com/hu guet-dictionnaire-du-xvie-s.html>.

62 C'était en tous cas valable au moment de la publication de l'article en anglais où j'ai pour la première fois indiqué ce sens ancien d'humanités, en juin 2017 (voir Clivaz, « Lost in translation »).

63 Le Deuff, Les humanités digitales, p. 35, cité p. 53 ci-dessus. Je reviendrai à cette proposition de Le Deuff (voir chapitre 3, p. 132-134).

64 Cormerais - Gilbert, «Introduction », p. 14 ; cité p. 55 ci-dessus.

65 M.-R. Moro, «L'infinie matière des humanités. Corps, espace, temps, au féminin pluriel », Cahiers de l'infantile 6 (2008), p. 119-129. 
en particulier, dans une acception qui rappelle le moule d'humanité, que nous avons relevé ci-dessus dans le contexte de la Renaissance.

Est-il possible de comprendre davantage cet oubli contemporain presque total du sens charnel et corporel d'humanité ? L'analyse foucaldienne du double tournant épistémologique de l'épistémè occidentale ${ }^{66}$ donne quelques pistes pour cartographier la question. A l'âge classique, dans l'édition préliminaire du Dictionnaire de l'Académie (1687), on constate à la fois l'émergence du sens académique des humanités et la disparition de sa signification charnelle : cette situation va perdurer dans les éditions suivantes. Au milieu du $19^{\mathrm{e}}$ siècle, deuxième tournant épistémologique souligné par Foucault, le langage prend sa revanche sur la catégorisation mise en place à lâge classique. Le sens charnel d'humanité trouve en effet refuge dans le Complément du dictionnaire de l'Académie publié en 1842 par Louis Barré qui définit ainsi le terme : «Humanité : Expr. prov., Reposer son humanité, Se mettre à son aise. // Avoir humanité, se disait autrefois pour, Être en vie. // Humanité, s'est dit Des parties sexuelles ${ }^{67}$.

On notera que dans sa définition, Barré dit clairement que les significations corporelles et sexuelles d'humanité étaient en usage autrefois, et se «sont dites », au passé. Il les mentionne néanmoins dans sa définition du dictionnaire complémentaire, qui est de fait un délicat exercice de communication académique. En effet, la sixième édition du Dictionnaire de l'Académie, en 1835, avait provoqué des polémiques, et Barré devra justifier de manière serrée l'édition d'un dictionnaire complémentaire, en tentant de ne pas froisser définitivement les autorités de l'Académie française ${ }^{68}$. On ne peut qu'être reconnaissant à Barré d'avoir gardé la mémoire dans ce dictionnaire complémentaire du sens incarné d'humanité(s). Sans doute sa fonction de professeur de philosophie lui donne ici un regard plus libre que celui du philologue, gardien des sens d'humanité(s) dans les éditions officielles du Dictionnaire de l'Académie. On perçoit en surface les profonds mouvements souterrains des plaques tectoniques des significations.

Il faut à ce stade tenter de considérer ensemble ces trois phénomènes : la presque disparition du sens charnel et corporel d'humanité(s), la fascination pour ce même élément incarné que d'aucuns perçoivent dans digital $(e)$, et la présence persistante et tenace d'humanités digitales en parallèle à humanités

66 Pour une présentation de cette thématique, voir Clivaz, L'ange et la sueur de sang, p. 99101.

67 L. Barré, « Humanité », « Humaniste », Complément du Dictionnaire de l'Académie, Paris: Firmin Didot frères, 1842, p. 586, <https://archive.org/details/fre_b1886109>.

68 Voir M. Marosvari, «Barré et la sixième édition du 'Dictionnaire de l'Académie' (18351842 ) , Romantisme 86 (1994), p. 89-100; ici p. 95; doi : 10.3406/roman.1994.5989, <http:// www.persee.fr/doc/roman_0048-8593_1994_num_24_86_5989>. 
numériques, malgré toutes les critiques essuyées, provincialisme inclus. Le passage au français de la part «intraduisible »- pour reprendre le terme de Derrida - du nom digital humanities révèle et rappelle à l'existence non seulement la formation aux langues classiques, du temps où on les "faisait», ces humanités, mais peut-être et surtout les liens profonds des humanités au corps et au corporel. On commence en conséquence à percevoir plus en profondeur les remarques épistémologiques qui ont été rassemblées dans l'introduction et le chapitre 1: Umberto Eco déclarant qu'internet n'est pas biologique ${ }^{69}$; Robert Darnton explicitant l'effet du smartphone par le terme Fingerspitzengefüh ${ }^{70}$, ou l'étude publiée dans Current Biology démontrant les effets de stimulation du smartphone sur le cerveau ${ }^{71}$.

Autour des significations d'humanités se rencontrent les sphères du corps et des technologies - ici digitales -, une interaction aussi vieille que l'humanité comme le rappelle François Jouen, directeur d'études à l'Ecole Pratique des Hautes Etudes : « comme les autres technologies avant elle, l'intelligence artificielle a déjà un impact sur le vivant [...]. Comme pour les inventeurs du silex taillé qui ont permis d'atteindre plus de proies, ce qui a augmenté la taille du cortex par l'apport de protéines. On est toujours dans ce même processus $»^{72}$. Avec toutefois une nuance de taille dans la rapidité de l'évolution de cette interaction : «Cette évolution conjointe peut encore une fois s'observer avec la surreprésentation du pouce dans le cortex des jeunes générations, qui est liée au fait qu'ils saisissent leurs messages avec ce doigt. Mais c'est vrai qu'aujourd'hui, on intègre des changements technologiques d'une génération à l'autre, ce qui est inédit ${ }^{73}$.

Pour sonder cet inédit, la prise en compte du langage - ici, le français - dans ses épaisseur et résistance historiques et épistémiques est nécessaire. Elle permet de saisir pourquoi il en va ainsi du corps dans la culture digitale, et ce dans le nom même des humanités digitales. C'est ce lieu du nom des humanités de plus en plus digitalisées que nous devons nous approprier pour surmonter l'oxymore si bien posé par Olender : les « documents matériels immatériels ${ }^{74}$. Le sentiment de déroute que nous éprouvons face au séisme du papier que l'on

\footnotetext{
69 Carrière - Eco, N'espérez pas, p. 22; cité dans l'Introduction, p. 3.

70 Darnton, The Case for Books, p. XIII ; cité dans l'Introduction, p. 5.

71 Gindrat et al., « Use-Dependent Cortical Processing »; cité dans l'Introduction, p. 4.

72 E. Cario, «L'intelligence artificielle a déjà un impact sur le vivant », Libération, 31 mai 2018, $<$ http://www.liberation.fr/debats/2018/05/31/l-intelligence-artificielle-a-deja-un-impactsur-le-vivant_1655682>.

73 Cario, «L'intelligence artificielle », <http://www.liberation.fr/debats/2018/05/31/l-intelligence-artificielle-a-deja-un-impact-sur-le-vivant_1655682>. Olender, Un fantôme, p. 28 ; cité au chapitre 1, p. 27.
} 
quitte, ou lorsque nous nous dégageons de cette matière «hylétique», selon les termes de Derrida ${ }^{75}$, trouve un lieu de transition dans la mémoire des humanités incarnées, de cette humanité corps, lieu de vie inclus, des parties sexuelles au ventre de la femme, selon Le dictionnaire de référence pour la langue de la Renaissance ${ }^{76}$.

Tourner notre attention davantage vers les humanités que vers leur adjectif numériques ou digitales trouve une légitimation supplémentaire dans l'observation de l'évolution linguistique de l'objet informatique digital computer, « ordinateur digital ». Il ne viendrait plus à l'idée de personne d'utiliser l'expression « ordinateur digital» (ou numérique), que nous ressentirions aujourd'hui comme redondante. Pourtant, pendant une quinzaine d'années, c'est bien ainsi qu'on a nommé ce nouvel objet, l'ordinateur numérique, digital computer. La première trace écrite de ce binôme, qui voulait se distinguer de l'ordinateur analogique, se trouve dans un rapport scientifique établi en 1942 par George Robert Stibitz, comme Bernard Williams l'a mis en évidence ${ }^{77}$. L'usage va demeurer bien quelques années: en 1950, l'article phare de Turing « Computing Machinery and Intelligence $»$ utilise couramment digital computer ${ }^{78}$.

A quel moment a-t-on cessé de parler de digital computer pour ne plus parler que d'ordinateur? De telles questions linguistiques restent souvent sans réponse claire, mais on peut se demander si une telle évolution n'attend pas aussi les humanités, qu'on les qualifie de numériques ou de digitales. A voir comme nous avons aujourd'hui absolument cessé de dire digital computer, il se pourrait bien, de manière similaire, que dans quelques années ou lustres, on parle simplement des humanités, forcément digitalisées, et qui auront fait leur transition depuis les sciences humaines via les humanités digitales. Cela dépendra bien sûr en bonne partie de la capacité des humanités à continuer à exister en tant que telles, sans être absorbées dans les études digitales.

Une première attestation du retour du sens générique des «humanités » ayant muté numériquement pourrait être le titre de cette nouvelle revue, dont le premier numéro a été publié en mai 2018, Les Cahiers de l'Agora: revue en

\footnotetext{
75 Derrida, « Le papier et moi », p. 46; cité au chapitre 1, p. 27.

76 Cité p. 56 ci-dessus.

77 Voir B. Williams, Computing with Electricity, 1935-1945, PhD Dissertation, University of Kansas, 1984; University Microfilms International, 1987; ici p. 310. Voir aussi la monographie de R. Dennhardt, The Term Digital Computer (Stibitz 1942) and the Flip-Flop (Turner 1920), Grin Verlag, 2016.

78 Voir par exemple Turing, «Computing Machinery and Intelligence», p. 50: « The reader must accept it as a fact that digital computers can be constructed, and indeed have been constructed, according to the principles we have described, and that they can in fact mimic the actions of a human computer very closely».
} 
humanités ${ }^{79}$. Ce premier numéro porte sur Ecritures contemporaines et processus de création, au sens tout à fait générique de ces termes. Mais les formes mêmes des articles - on trouve entre autres une vidéo - attestent de la mutation numérique de ces écritures, que l'introduction reconnaît explicitement : « la dimension réflexive des écritures contemporaines n’est pas indépendante des supports et l'on peut penser qu'elle est largement favorisée par le numérique qui offre aux écrivains des espaces de réflexivité plus libres ${ }^{80}$. La mue numérique pourrait bien rendre les sciences humaines à leurs humanités, et cet ouvrage espère démontrer la densité de l'héritage et la force de signification que véhiculent les humanités, une densité et un héritage à mettre en jeu dans le dialogue interdisciplinaire stimulé par la culture digitale.

\section{Deuxième partie : Lire Alan Turing et considérer le rôle de l'esprit dans les digital humanities}

\subsection{Introduction ${ }^{81}$}

Dans cette seconde partie du chapitre 2 , je souhaite mettre en œuvre la proposition de Milad Doueihi à laquelle il est fait référence en préambule de ce chapitre, soit «proposer une autre généalogie aux humanités numériques, en relisant « Computing Machinery and Intelligence » d'Alan Turing pour retrouver une partie importante des questionnements épistémologiques et philosophiques, voire même méthodologiques, qui sont toujours d'une grande actualité ${ }^{82}$. L'article de Turing est une mine d'or destinée à être encore longtemps exploitée, en particulier dans le sens indiqué par Doueihi. Pour ce qui nous concerne, nous allons scruter les usages et la mise en scène du terme « esprit »- mind sous la plume de Turing. En préambule de ce chapitre, nous

79 Voir Les Cahiers de l'Agora : revue en humanités, <https://www.u-cergy.fr/fr/laboratoires/ agora/cahiers-d-agora.html>.

80 V. Houdart-Merot - A.-M. Petitjean, « Introduction. Réfléchir l'engagement dans l'écriture aujourd'hui : acteurs, lieux et enjeux », Les Cahiers de l'Agora : revue en humanités 1 (2018), $<$ https://www.u-cergy.fr/fr/laboratoires/agora/cahiers-d-agora/numero-1/introduction. html >.

81 Cette seconde partie de chapitre traduit et développe un article publié en anglais, avec l'aimable autorisation des éditeurs : Clivaz, C., « Thinking About the 'Mind' in Digital Humanities : Apple, Turing and Lovelace », dans Information technology in the humanities. International scientific and practical conference, Krasnoyarsk 18-22 septembre 2017, Siberian Federal University (éd.), SFU, 2018, p. 5-14 ; <http://lib3.sfu-kras.ru/ft/LIB2/ELIB/b71/ free/i-838981639.pdf>.

82 Doueihi, «Quête et enquête », p. 8-9; il est fait référence à ce passage au chapitre 1, p. 31 ; il est cité en préambule de ce chapitre 2, p. 44. 
avons vu en effet qu'au moins deux langues, l'hébreu et l'allemand, se réfèrent à l'esprit en traduisant le nom des digital humanities ${ }^{83}$.

Alan Turing a publié en 1950 cet article consacré à l'informatique et à l'épistémologie dans le journal Mind, fondé en 1876 et réputé d'abord dans le domaine de la philosophie, puis s'ouvrant de plus en plus au dialogue interdisciplinaire. En soumettant son article à Mind, Turing montre son attention à ce dialogue, une préoccupation qui l'accompagnera tout au long de ses recherches, qui le conduiront jusque dans le domaine de la mathématique biologique. Il publiera en effet en 1952 un article consacré à la morphogenèse, « The Chemical Basis of Morphogenesis », qui fait date également ${ }^{84}$. Le ton épistémologique de son article « Computing Machinery and Intelligence » conduit parfois certains auteurs, comme Darren Abramson, à se sentir le devoir de défendre Turing : celui-ci s'éloignerait trop, dans « Computing Machinery », d'arguments mathématiques au profit d'un raisonnement philosophique ${ }^{85}$. De fait, Abramson met en lumière comment Turing y soutient bel et bien une thèse philosophique, sur la nature de l'esprit (mind) humain ${ }^{86}$. Il est donc judicieux de pister cette thématique dans cet article. Comme signalé, Turing nomme digital computer le nouvel ordinateur, dont il commente ainsi l'impact :

Mon intérêt présent à « penser les machines » a été suscité par une sorte particulière de machine, désignée habituellement comme un « ordinateur électronique » ou un « ordinateur digital ». En suivant cette suggestion, nous donnons la permission aux ordinateurs digitaux de prendre part à notre jeu ${ }^{87}$.

Il décrit l'ordinateur numérique idéal comme un « ordinateur humain » :

\footnotetext{
83 Voir p. $44-45$ ci-dessus.

84 A. Turing, «The Chemical Basis of Morphogenesis », Philosophical Transactions of the Royal Society of London B, 237 (1952), p. 37-72, <https://doi.org/10.1098/rstb.1952.0012>.

85 D. Abramson, « Turing's Responses to Two Objections », Minds and Machines 18 (2008/2), p. $147-167$.

86 Abramson, «Turing's Responses », p. 147: «I argue that by understanding Turing's responses to these objections more clearly, we discover a hitherto unrecognized, substantive thesis in his philosophical thinking about the nature of mind ».

87 Turing, « Computing Machinery », p. $436:$ «the present interest in 'thinking machines' has been aroused by a particular kind of machine, usually called an 'electronic computer' or 'digital computer'. Following this suggestion we only permit digital computers to take part in our game».
} 
Ce qui se tient derrière les ordinateurs digitaux peut être expliqué ainsi : ces machines sont censées pouvoir faire toute opération qu'un ordinateur humain pourrait mener à bien. L'ordinateur humain est censé suivre des règles fixes : il n'a pas l'autorité nécessaire pour les modifier en aucun point. Nous pourrions imaginer que ces règles sont écrites dans un livre, qui se modifie à chaque fois que l'humain est affecté à une nouvelle tâche. Il peut bénéficier d'une quantité infinie de papier pour faire ses calculs. Il a aussi la possibilité de faire ses multiplications et additions sur un «bureau machine », mais ce n'est pas important ${ }^{88}$.

Pour entrouvrir la possibilité, pour cette machine décrite comme un « ordinateur humain », de réellement penser, Turing explique ensuite qu'il convient d'y ajouter un élément de hasard ${ }^{89}$. Cet élément de hasard et de libre volonté seraient ce qui est nécessaire pour «laisser penser » la machine. Des six objections soulevées dans son article à l'encontre de l'idée qu'une machine pourrait penser, Turing met une énergie particulière à contrer celle qu'il estime la plus ferme, émise par Ada Lovelace, au siècle précédent ${ }^{90}$. Comme il le résume,

l'information très détaillée dont nous disposons sur l'engin analytique de Babbage provient du mémoire de Lady Lovelace (1842). Elle y affirme que « l'engin analytique n'a pas prétention à être l'origine de quelque chose. Il peut faire tout ce que nous sommes capables de lui ordonner de performer $\gg(\text { ses italiques). Cette affirmation est citée par Hartree (1949) })^{91}$.

88 Turing, « Computing Machinery », p. 436 : « The idea behind digital computers may be explained by saying that these machines are intended to carry out any operations which could be done by a human computer. The human computer is supposed to be following fixed rules; he has no authority to deviate from them in any detail. We may suppose that these rules are supplied in a book, which is altered whenever he is put on to a new job. He has also an unlimited supply of paper on which he does his calculations. He may also do his multiplications and additions on a 'desk machine', but this is not important ».

89 Turing, « Computing Machinery », p. 438.

90 Abramson, «Turing's Responses », p. 157.

91 Turing, « Computing Machinery », p. $447:$ « Our most detailed information of Babbage's Analytical Engine comes from a memoir by Lady Lovelace (1842). In it she states, 'The Analytical Engine has no pretensions to originate anything. It can do whatever we know how to order it to perform' (her italics). This statement is quoted by Hartree (1949) ». Turing se réfère ici à D. R. Hartree, Calculating Instruments and Machines. University of Illinois Press, 1949 . 
Valeria Aurora a souligné, dans sa défense d'Ada Lovelace, que Turing l'avait mal lue, alors qu'elle défendait un point de vue similaire au sien ${ }^{92}$. On arrive ici à une question complexe et passionnante: comment les chercheurs d'aujourd'hui évaluent-ils le rapport d'un pair en 1950 à une intellectuelle du milieu du $19^{e}$ siècle ? La question est tout sauf anecdotique, nous allons le voir, et donne l'itinéraire de cette deuxième partie de chapitre : elle revient sur le texte d'Ada Lovelace et sur l'écrit de Louis-Frédéric Menabrea qu'elle traduit ; la troisième partie présentera une réflexion à propos de la double désignation en anglais de l'esprit, mind et spirit, et de ce qui n'est pas pensé (unthought); enfin la quatrième partie donnera le mot de la fin de ce chapitre portant sur les humanités digitales et leurs appellations.

\section{2 (Re)lire Ada Lovelace en 1950 et aujourd'hui}

La figure d'Ada Lovelace ne manque pas d'interroger, et fait l'objet de diverses évaluations. Pour Valeria Aurora, co-fondatrice de l'Ada Initiative ${ }^{93}$, « elle est la première programmatrice au monde d'un software en open source, car elle a publié la source de sa programmation ${ }^{94}$. Tandis que Thomas Misa considère que cette affirmation "pourrait faire l'objet d'une évaluation pondérée», quand bien même on peut attribuer à Lovelace l'auctorialité du « premier algorithme conçu pour une machine informatique $»^{95}$. On devine qu'en un $21^{\mathrm{e}}$ siècle où se référer à des figures historiques féminines est devenu un besoin identitaire vital, la polémique ne se laissera pas aisément résoudre ${ }^{96}$. On peut toutefois faire œuvre utile en allant regarder de près ce qu'a dit l'intéressée, qui se montre absolument consciente des différents enjeux de la problématique discutée par Turing, comme nous allons le voir.

Le dossier réserve une première surprise de taille : peu de chercheurs semblent être allés lire réellement Ada Lovelace, alors même que son œuvre, ap-

92 V. Aurora, « Rebooting the Ada Lovelace Mythos », dans Ada's Legacy. Cultures of Computing from the Victorian to the Digital Age, R. Hammerman - A. L. Russell (éd.), ACм Publishers, 2016, p. 231-239; ici p. 232-233.

93 Aurora, «Rebooting», p. 236. L’entreprise s'est terminée en 2015 (voir <https://adainitia tive.org/about-us/>).

94 Aurora, «Rebooting», p. 236 : «she's the world's first open source software programmer because she published the source to her program ».

95 T. J. Misa, « Charles Babbage, Ada Lovelace, and the Bernoulli Numbers », dans Ada's Legacy. Cultures of Computing from the Victorian to the Digital Age, R. Hammerman - A. L. Russell (éd.), ACM Publishers, 2016, p. 11-31; ici p. 12.

96 Pour Allan Bromley, Lovelace n’a rien programmé du tout (Misa, « Charles Babbage », p. 16). 
partenant au domaine public, est disponible sur Google Books ${ }^{97}$. Lovelace a produit une traduction en anglais de vingt-cinq pages du traité de Louis-Frédéric Menabrea sur l'engin analytique de Charles Babbage, publié en 1842 en français ${ }^{98}$. Elle le complètera de quarante-quatre pages de notes avec un tableau conséquent sur les nombres de Bernoulli, le tout publié en $1843^{99}$. Sur la couverture de l'ouvrage elle reste anonyme, se désignant comme the translator, et ajoute dans les notes ci et là ses initiales « A. L. L. ». Autrement dit, elle tire prétexte de cette traduction pour se donner le droit d'écrire aussi. Afin de rendre justice à la plume d'auteur de Lovelace, j'ai fait le choix d'indiquer séparément dans la bibliographie sa traduction de Menabrea et ses propres notes.

Valeria Aurora pense certainement bien faire en volant au secours d'Ada Lovelace, qui, selon elle, serait injustement mécomprise par Alan Turing ${ }^{100}$. Pour ce faire, elle met en route un raisonnement compliqué, qui de fait, après vérification, ne se trouve pas dans le texte de Lovelace $^{101}$. Je doute honnêtement qu'elle ait été le lire : elle ne renvoie jamais à la pagination du texte de Lovelace. Elle admet aussi n'avoir aucune idée de la manière dont Turing aurait eu accès à ce texte ${ }^{102}$. En scrutant de près l'article de Turing et d'autres sources, il me semble pouvoir affirmer qu'il ne se réfère pas directement au texte d'Ada Lovelace, mais s'appuie sur la médiatisation qu'en fait Douglas Rayner Hartree en

97 Voici le lien qui y conduit: $<$ https://books.google.ch/books?id=EIVqHUmgWlkC\&printse $\mathrm{c}=$ frontcover\&hl=fr\&source=gbs_ge_summary_r\&cad=0 $\# \mathrm{v}=$ onepage\& $\mathrm{q} \& \mathrm{f}=$ false $>$.

98 F. L. Menabrea, Sketch of the Analytical Engine Invented by Charles Babbage, extracted from the Scientific Memoirs vol. 3, Ada Lovelace (trad.), London : Richard \& Taylor, 1843, p. 666-69o.

99 A. Lovelace, Notes on Menabrea's Sketch of the Analytical Engine Invented by Charles Babbage, extracted from the Scientific Memoirs, vol. 3, London : Richard \& Taylor, 1843, p. 691731 \& $73^{2-735}$ (1 à 4$)$.

100 Aurora, «Rebooting », p. $232:$ «He calls one of these arguments 'Lady Lovelace's Objection', which I think is unfair because he misinterprets what she's trying to say to make his point ».

101 Aurora, « Rebooting », p. 232 : « Lovelace in the Notes tried to counteract a particular misconception that was common in her time. People would say, 'This thing just calculated the answer to $3^{+2}$. It must be living !' In particular, people would ask, 'What if I tell it the wrong question? Will it still give me the right answer?' People had no idea how computers worked. She was trying to explain that these machines can only do what you tell them too ; somebody still has to come up with the program, encode it, and stick it in the machine. Turing interpreted her statement about this as 'machines can never surprise you' and went on to demonstrate that machines could indeed surprise you with their results - but that's not what Ada was saying at all. At least Turing got her name back in circulation».

102 Aurora, «Rebooting», p. 233: «I have no idea how he became aware of her. Perhaps everyone passed the Notes around at Cambridge or something. I would love to know ». 
1949 : on a vu ci-dessus que c'était le cas dans l'article de $1950^{103}$, et c'est ce qu'il répète dans une émission radiodiffusée en 1951 sur la В ВC, « Can Digital Computers think ? »104. L'enregistrement original a disparu ${ }^{105}$, mais la transcription de l'émission est disponible en ligne ; elle confirme on ne peut plus clairement l'importance de « l'objection Lovelace » pour Turing, qui démarre d'emblée sur cette thématique ${ }^{106}$.

Last but not least, en amont de ces deux références à Hartree, on a une première mention par Turing de cette objection sans la référence à Lovelace, dans une formulation indirecte, en 1947, soit avant la publication de l'ouvrage de Hartree : « on a dit que les machines ordinateurs pouvaient seulement mener à terme les processus pour lesquels on les avait programmées » ${ }^{107}$. L'objection semble donc courir dans la période d'après-guerre, sans être toujours liée au nom d'Ada Lovelace : Hartree, puis Turing lui rendront cette justice, mais celuici ne semble pas avoir pour autant ouvert les notes de Lovelace. Il aurait pourtant gagné à le faire, car son interlocutrice du siècle précédent montre qu'elle a conscience de la complexité de la question.

Relire Turing, puis Lovelace et Menabrea avant elle, permet une intéressante généalogie de la question de l'esprit confronté à l'informatique. Ou, pour reprendre la phrase d'Ada passée à la postérité via Turing, la question de savoir si un ordinateur analogique peut avoir une prétention à créer quelque chose, à être à l'origine de quelque chose : «L'Engin Analytique n'a aucune prétention à être à l'origine (to originate) de quoi que ce soit »108. Comme nous venons de le constater, Turing en reste à cet adage sans prendre en compte son contexte et

103 Turing, « Computing Machinery », p. 447.

104 A. Turing, « Can digital computers think? », TS with AMS annotations of a talk broadcast on ввс Third Programme, 15 May 1951, transcribed on <http://www.turingarchive.org/ browse.php/B/5>, p. 1-8; ici p. 2.

105 Voir Alan Turing's lost radio broadcast rerecorded. 15th of May 1951, BBC < https://www.you tube.com/watch?v=cMxbSsRntv4> ; la lecture de la conférence de Turing a été enregistrée. Au début de cette vidéo, on apprend qu'aucun enregistrement de Turing ne nous est parvenu, apparemment : «Unfortunately, this recording no longer exist, along with all other recordings of Alan Turing» (0:36-0:41).

106 Turing, « Can digital computers think? », <http://www.turingarchive.org/browse.php/B/ $5>$; ici p. 1.

107 A. Turing, «Lecture on the automatic computing engine » (1947), dans The Essential Turing, B. J. Copeland (éd.), Oxford University Press, 2004, p. 392: « It has been said that computing machines can only carry out the processes that they are instructed to do »; cité par Abramson, « Turing's Responses », p. 158. David De Roure, directeur de l'Oxford e-Research Center et spécialiste de Turing, m’a confirmé par oral qu'à sa connaissance aussi, Turing ne semble pas avoir lu directement Lovelace.

108 Lovelace, Notes on Menabrea's Sketch, p. 722: « The Analytical Engine has no pretensions whatever to originate anything ». 
les autres nuances faites par Lovelace. Ce qu'il exprime donc dans son article de $195^{\circ}$ et l'émission ввС de $195^{1}$ est à évaluer en fonction de cet adage, et non pas comme écho à la position d'Ada Lovelace. Turing développe deux arguments principaux pour contrer ce qu'il nomme l'« objection de Lovelace». Le premier est l'élément du hasard, mais qu'il verbalise plus exactement comme l'effet de surprise, dans la citation ci-dessous. Il y évoque les ordinateurs numériques sur un mode anthropomorphique :

Une meilleure variante de cette objection postule qu'une machine ne peut jamais nous «prendre par surprise ». Cette affirmation est un défi plus direct et peut être adressée de manière directe. Les machines me prennent par surprise très fréquemment. Cela est largement dû au fait que je ne fais pas assez de calculs pour décider de ce que je pourrais attendre d'eux, ou plutôt, cela est largement dû au fait que, bien que je calcule, je le fais en hâte, d'une manière peu soignée, en prenant des risques ${ }^{109}$.

Pour reconnaître à la machine la possibilité de créer quelque chose qui soit une surprise pour l'humain, Turing doit évoquer les moments où son propre esprit montre des signes d'inefficacité : « ... bien que je calcule, je le fais en hâte, d'une manière peu soignée, en prenant des risques ». C'est dans cet aveu des limites de l'esprit humain que se tient le tournant de l'argumentation de Turing : il reconnait ainsi implicitement une concurrence entre l'esprit humain et le potentiel informatique, une reconnaissance devenue aujourd'hui explicite et de plus en plus vivement débattue. En 1950, Turing tourne déjà ses regards vers le futur en évoquant les développements à venir de l'informatique. Revenant au terme de son article à l'objection de Lovelace, comme la plus significative à discuter, il ne peut donner comme argument conclusif qu'un appel au futur :

Revenons-en pour l'instant à l'affirmation de Lady Lovelace qui soutenait que la machine ne peut faire que ce que nous lui disons de faire. [...] Le seul appui réellement satisfaisant qui puisse être donné à cette vision

109 Turing, « Computing Machinery », p. 448: «A better variant of the objection says that a machine can never 'take us by surprise'. This statement is a more direct challenge and can be met directly. Machines take me by surprise with great frequency. This is largely because I do not do sufficient calculation to decide what to expect them to do, or rather because, although I do a calculation, I do it in a hurried, slipshod fashion, taking risks ». 
sera fourni par l'attente de la fin du siècle, en faisant alors l'expérience décrite ${ }^{110}$.

C'est ici qu'il est important d'aller relire Lovelace elle-même. En effet, de la même manière que le fait Turing, elle pose comme point de fuite aux limites du raisonnement ce qui pourra être expérimenté, découvert : « Pour en revenir aux facultés d'action de cet engin : la question doit surgir dans tout esprit (mind), sont-elles réellement capables de suivre l'analyse dans toute son extension? Aucune réponse, entièrement satisfaisante pour tous les esprits, ne peut être donnée à cette interrogation, à part l'existence concrète de l'engin, ainsi que l'expérimentation concrète de ses résultats pratiques »"111. Comme on le voit, Ada Lovelace est loin d'avoir une position univoque et close sur la fameuse « objection », et il est intéressant de voir qu'elle pose, comme Turing, le critère des expériences à venir pour y répondre réellement. L'un et l'autre appliquent clairement ce que nous reconnaissons comme le critère scientifique de l'expérimentation pour vérifier les dires.

Pour continuer à rendre justice à Ada Lovelace, je citerai encore ce passage, qui précède immédiatement son célèbre adage « the Analytical Engine has no pretensions whatever to originate anything »: «En considérant tout nouveau sujet, on a souvent tendance, d'une part, à surestimer ce qu'on trouve d'emblée intéressant ou remarquable; et, d'autre part, par une sorte de réaction naturelle, [on a aussi tendance] à sous-estimer le véritable état de la question, lorsque nous constatons que nos notions ont dépassé celles qui étaient réellement acceptables ${ }^{112}$. Ces phrases montrent combien Ada Lovelace est circonspecte dans son évaluation de la question qu'elle termine, on l'a vu, par l'appel à la vérification par l'expérience. On ne peut donc pas conclure avec

110 Turing, «Computing Machinery and Intelligence», p. 452 et 455 : « Let us return for a moment to Lady Lovelace's objection, which stated that the machine can only do what we tell it to do. [...] The only really satisfactory support that can be given for the view expressed will be that provided by waiting for the end of the century and then doing the experiment described ».

111 Lovelace, Notes on Menabrea's Sketch, p. 723 : « To return to the executive faculties of this engine : the question must arise in every mind, are they really even able to follow analysis in its whole extent ? No reply, entirely satisfactory to all minds, can be given to this query, excepting the actual existence of the engine, and actual experience of its practical results ».

112 Lovelace, Notes on Menabrea's Sketch, p. $722:$ «In considering any new subject, there is frequently a tendency, first, to overrate what we find to be already interesting or remarkable ; and, secondly, by a sort of natural reaction, to undervalue the true state of the case, when we do discover that our notions have surpassed those that were really tenable ». 
Bernhardt que son erreur aurait été de surestimer l'esprit humain ${ }^{113}$ : elle apparaît même plus circonspecte que Menabrea qu'elle commente, comme on le verra ci-dessous.

On ne trouve pas non plus trace dans son écrit de réactions de personnes naïves auxquelles elle chercherait à donner un cadre plus rationnel, comme le supposait Valeria Aurora ${ }^{114}$. C'est bien avec la vision de Louis-Frédéric Menabrea qu'Ada Lovelace en découd, et il vaut la peine de remonter à ce qui est, finalement, la source de ce débat autour de la capacité d'un ordinateur à penser, jusque chez Turing, soit Menabrea. Décrivant l'Engin Analytique de Babbage, premier ancêtre de l'ordinateur, Menabrea pointe d'entrée de texte sur la question de l'esprit :

Les travaux qui appartiennent à plusieurs branches des sciences mathématiques, quoique paraissant, au premier abord, être uniquement du ressort de l'esprit, peuvent néanmoins se diviser en deux parties distinctes : l'une qu'on peut appeler mécanique, parce qu'elle est sujette à des lois précises et invariables, susceptibles d'être traduites physiquement, tandis que l'autre, qui exige l'intervention du raisonnement, est plus spécialement du domaine de la pensée. Dès lors on pourra se proposer de faire exécuter par le moyen de machines la partie mécanique du travail, et réserver à la seule intelligence celle qui dépend de la faculté de raisonner ${ }^{115}$.

On est ici à la racine historique des réactions documentées devant l'Engin Analytique ${ }^{116}$. Menabrea commence par situer l'intégralité de la problématique sous la bannière de l'esprit, puis se ravise en un deuxième temps. Il pose alors

113 C. Bernhardt, Turing's vision. The Birth of Computer Science, Mit Press, 2016, p. 70 : « As David Deutsch points out, the error that Lovelace made was really not in underestimating computers, but in overestimating the human brain at a time when many supernatural beliefs were commonly held ».

114 Voir Aurora, « Rebooting », p. 232 ; cité en note, p. 65 ci-dessus.

115 F. L. Menabrea, « Notions sur la Machine Analytique de M. Charles Babbage », Bibliothèque Universelle de Genève 41, Paris : Chez Anselin, 1842, p. 352-376 ; ici p. $35^{2}$; <http:// www.bibnum.education.fr/calcul-informatique/calcul/notions-sur-la-machine-analy tique-de-m-charles-babbage >.

116 Voici comment Menabrea décrit cet engin : «M. Charles Babbage a consacré plusieurs années à réaliser une pensée gigantesque. Il ne s'est proposé rien moins que de construire une machine capable d'exécuter, non seulement les calculs arithmétiques ; mais encore les calculs analytiques, dont les lois seraient connues »; « Mr. Babbage pense pouvoir, par le moyen de sa machine, faire en trois minutes le produit de deux nombres composés de vingt chiffres chacun » (Menabrea, « Notions sur la Machine Analytique», p. 353 et 373). 
la division entre les opérations «purement mécaniques » et ce qui demanderait de «l'intelligence », ou « de la faculté de raisonner». Il exprime cette dichotomie de différentes manières dans son texte, jusqu'à poser cette affirmation qu'Ada Lovelace ne fera que reformuler dans sa célèbre « objection », en note de ce passage de Menabrea : « l'interprétation des formules et des résultats est en dehors des attributions [de la machine], à moins toutefois que cette interprétation ne soit elle-même susceptible d'être exprimée par le moyen des symboles dont elle fait usage. Ainsi elle n'est point elle-même l'être qui pense, mais on peut la considérer comme l'être qui exécute les conceptions de l'intelligence $»^{117}$.

On a ici la source directe de l'objection de Lovelace, qu'il faudrait désormais bien plutôt nommer l'objection de Menabrea, et qui repose sur la dichotomie qu'il a posée en tout début de texte, non sans avoir brièvement commencé par dire que la problématique ressortit à l'esprit. A mon avis, c'est exactement là que se tient le nœud de l'affaire, et ce sur quoi nous avons à méditer aujourd'hui face à l'intelligence artificielle. Cette dichotomie est reprise par Turing dans ce passage de fin d'article où il revient sur l'objection de Lovelace :

En considérant les fonctions de l'esprit ou du cerveau (of the mind or the brain), nous trouvons certaines opérations que nous pouvons expliquer en termes purement mécaniques. Mais ce que nous disons là ne correspond pas à l'esprit réel : c'est une sorte de peau, que nous devons retirer pour trouver l'esprit réel. Mais ensuite, dans ce qui reste, nous trouvons une autre peau à retirer, et ainsi de suite. En procédant de cette manière, avons-nous une chance de jamais parvenir à l'esprit réel, ou arriveronsnous finalement à la peau qui n'a plus rien en elle? Dans ce dernier cas de figure, l'esprit serait intégralement mécanique ${ }^{118}$.

Turing réduit de fait la dichotomie de Menabrea au pôle mécanique et aligne l'esprit sur le cerveau, tout en laissant de l'espace au questionnement en finale. Cette attitude est en accord avec le fait qu'il balaie rapidement l'objection théologique : Dieu n'aurait donné une âme qu'aux hommes. Turing répond à

\footnotetext{
117 Menabrea, « Notions sur la Machine Analytique », p. 375.

118 Turing, « Computing Machinery and Intelligence», p. 454-455: «In considering the functions of the mind or the brain, we find certain operations which we can explain in purely mechanical terms. This we say does not correspond to the real mind: it is a sort of skin, which we must strip off if we are to find the real mind. But then in what remains we find a further skin to be stripped off, and so on. Proceeding in this way do we ever come to the "real" mind, or do we eventually come to the skin, which has nothing in it? In the latter case the whole mind is mechanical».
} 
cette objection en soulignant qu'il y a moins de différence entre un homme et un animal, qu'entre un animal et un objet inanimél19, amenant ainsi de l'eau au moulin de l'anti-spécisme avant l'heure ${ }^{120}$, une thématique bien présente aujourd'hui. Mais si, dans « Computing Machinery and Intelligence », la position de Turing paraît claire - le mind n'est sans doute qu'un brain, et la distinction entre capacités mécaniques et interprétatives ou intellectuelles s'estompe une telle conception semble par bien des aspects l'avoir emporté puisqu'un très important projet de recherche européen se nomme le Human Brain project $t^{121}$. Dans la mesure où Yves Citton a posé en 2010 l'interprétation comme savoir-faire central des sciences humaines ${ }^{122}$, on voit bien qu'on touche ici à un point névralgique de notre regard sur l'intelligence artificielle ; c'est aussi l'interprétation que Menabrea soulignait comme lieu de la différence entre l'esprit humain et la machine. Mais gardons-nous de simplifier la pensée de Turing et de forcer le trait, car il apporte lui-même une dimension plus profonde encore à son point de vue dans la conférence radiophonique qu'il donne en 1951 à la BBC.

Sa position quant à l'« objection Lovelace» se renforce encore, mais il y ajoute ici la notion de «mystère », ce qui peut surprendre au premier abord, mais donne précisément son ampleur de champ à la conclusion de cette conférence « Can Digital Computers Think ?» :

J'espère certainement et crois qu'aucun effort de taille ne sera consacré à la confection de machines avec les traits les plus distinctivement humains mais non intellectuels; comme par exemple la forme d'un corps humain ; car c'est à mes yeux plutôt futile de conduire de telles expériences, et leurs résultats auraient quelque chose de la qualité déplaisante

119 Turing, « Computing Machinery and Intelligence », p. 443 : « God has given an immortal soul to every man and woman, but not to any other animal or to machines. Hence no animal or machine can think. I am unable to accept any part of this, but will attempt to reply in theological terms. I should find the argument more convincing if animals were classed with men, for there is a greater difference, to my mind, between the typical animate and the inanimate than there is between man and the other animals ».

120 L'un des ouvrages initiaux de l'anti-spécisme est Animal Liberation de Peter Sinner (Harper Collins, 1975). Pour un état des lieux récent sur l'animal du point de vue théologique et en regard du questionnement anti-spéciste, voir M. Cutino, I. Iribarren et F. Vinel (éd.), $L a$ restauration de la création. Quelle place pour les animaux? Actes du colloque de l'ERCAM tenu à Strasbourg du 12 au 14 mars 2015, Brill, 2018.

121 Voir Human Brain Project, <https://www.humanbrainproject.eu/en/>.

122 Citton, L'avenir des humanités, édition Kindle, l. 407 : « Autant que de l'économie des connaissances, l'avenir de ces humanités dépend des cultures de l'interprétation qu'elles parviendront (ou non) à vivifier, afin de cultiver à la fois leur pluralité et leurs solidarités ». 
des fleurs artificielles. Les expériences visant à produire une machine qui pense me semble appartenir à une autre catégorie. L'ensemble du processus de pensée est encore bien mystérieux pour nous, mais je crois que tenter l'expérience de fabriquer une machine pensante nous aidera grandement à découvrir comment nous pensons nous-mêmes ${ }^{123}$.

Ces derniers propos de Turing permettent de nouer la gerbe de cette section, qui a mis en évidence la généalogie du questionnement autour de l'esprit et de la reproductibilité de la pensée informatique, exprimé par Louis-Frédéric Menabrea, approfondi et affiné par Ada Lovelace, et développé avec maestria par Alan Turing. Celui-ci dit l'intérêt de la quête de l'intelligence artificielle pour mieux comprendre notre propre manière de penser, plus que pour la supplanter. C'est qu'il en va, selon ses mots, de «l'ensemble du processus de pensée [...] encore bien mystérieux pour nous ». Près de septante ans plus tard, François Jouen exprime un point de vue similaire : «le jour où on aura des systèmes d'intelligence artificielle qui commenceront à avoir des propriétés relativement proches du fonctionnement humain - dont on ne connaît finalement pas grand-chose - on le comprendra sans doute mieux ${ }^{124}$.

Mais à cet approfondissement analogique de la comparaison des systèmes de cognition, Jouen ajoute de manière cruciale des éléments de dissemblance entre IA et cerveau humain, car « les systèmes d'IA ne possèdent pas les capacités génératives liées à l'apprentissage ${ }^{125}$. Ainsi, s'il estime évident qu'un jour «les machines penseront», il précise aussi qu'il faudra compter sur la différenciation des modes de pensée entre humain et IA : «sur le plan de la pensée, c'est plus simple de chercher à reproduire le fonctionnement humain. Je suis cependant persuadé que dans quelque temps, on aura des systèmes cognitifs artificiels qui auront des propriétés radicalement différentes » ${ }^{126}$.

123 Turing, «Can Digital Computers Think ?», p. 8: «I certainly hope and believe that no great efforts will be put into making machines with the most distinctively human, but non-intellectual characteristics ; such as the shape of a human body ; for it appears to me to quite futile to make such attempts and their results would have something like the unpleasant quality of artificial flowers. Attempts to produce a thinking machine seem to me to be in a different category. The whole thinking process is still rather mysterious to us, but I believe that the attempt to make a thinking machine will help us greatly in finding out how we think ourselves ».

Cario, «L'intelligence artificielle», <http://www.liberation.fr/debats/2018/05/31/lintelligence-artificielle-a-deja-un-impact-sur-le-vivant_1655682>.

125 Ibid.

126 Ibid. 
Cette dernière réflexion sur la différenciation qui s'annonce me paraît capitale : à voir les efforts investis dans la construction du très mimétique robot japonais Erica, pressenti pour présenter le journal télévisée ${ }^{27}$, il faut espérer que les propos de Jouen soient entendus par bon nombre de ses collègues, afin qu'ils se préparent à percevoir ces «propriétés radicalement différentes » de l'IA. Un regard issu des sciences humaines peut ici apporter sa contribution en s'attachant aux noms et aux mots, selon le projet de ce chapitre, pour mettre en évidence la complexité des processus de pensée et de la notion d'esprit.

\subsection{Mind, brain, spirit, unthought: décliner les noms de l'esprit}

La mention par Turing de l'aspect «mystérieux » du processus de pensée, dans la conclusion de la conférence de 1951, surprend d'autant plus que dans « Computing Machinery and Intelligence », il n'ouvrait pas ce registre. Il n'y utilisait pas non plus le terme de spirit, mais seulement ceux de mind et brain, en finissant par aligner le plus possible mind sur brain, comme nous l'avons vu. L'anglais permet la dichotomie entre l'esprit-mind et l'esprit-spirit, ce qui n'est ni le cas du français, ni celui de l'allemand qui entend donc les deux sens dans Geist et dans digitaler Geist. L'absence de l'esprit frappe d'autant plus, si on compare l'article de Turing avec celui, non moins célèbre, de Vannevar Bush, publié juste cinq ans plus tôt : «As We May Think » ${ }^{128}$. Bush cherche à articuler capacités de la machine et mind, tout en gardant à ce dernier sa spécificité :

L'esprit humain opère par association. [...] La sélection par association, plutôt que par indexation, pourrait être mécanisée. On ne peut pas espérer égaler la rapidité et la flexibilité avec lesquelles l'esprit suit un chemin associatif, mais il devrait être possible de battre l'esprit de manière décisive en ce qui concerne la permanence et la clarté des items ressuscités à partir de lieux de stockage ${ }^{129}$.

\footnotetext{
127 Voir Radio Canada, «Au Japon, le téléjournal est présenté par un robot nommé Erica », $<$ http://ici.radio-canada.ca/nouvelle/1082408/robot-humanoide-erica-japon-journaltelevision-intelligence-artificielle >.

128 Bush, « As We May Think », 1945 .

129 Bush, «As We May Think », 1945, p. $6:$ : The human mind operates by association. [...] Selection by association, rather than indexing, may yet be mechanized. One cannot hope thus to equal the speed and flexibility with which the mind follows an associative trail, but it should be possible to beat the mind decisively in regard to the permanence and clarity of the items resurrected from storage ».
} 
Il n'aligne donc pas l'esprit-mind sur le cerveau. Alors même qu'il pose dans cet article les bases impressionnantes du Memex ${ }^{130}$, Bush inscrit toute sa réflexion dans une perspective éthique et de conscientisation de ses contemporains, au lendemain de l'armistice de la Seconde Guerre mondiale. C'est dans sa conclusion qu'il utilise une fois, mais avec une certaine emphase, le terme esprit/ spirit :

On peut présumer que l'esprit (spirit) de l'homme devrait être élevé s'il peut avoir une meilleure vision de son passé fait d'ombres, et analyser plus complètement et objectivement ses problèmes présents. Il a construit une civilisation si complexe qu'il a besoin de mécaniser ses enregistrements [...], en dépassant sa mémoire limitée ${ }^{131}$.

On peut dire que Vannevar Bush fait usage du terme spirit dans le cadre de la réflexion sur la norme éthique et philosophique, pour désigner l'esprit sous son aspect de catégorie générale et générique. C'est un registre différent de celui de « mystère », évoqué par Turing pour dire les limites du savoir présent, en fin de conférence. Il vaut donc la peine de s'interroger sur l'absence du terme spirit dans l'article-clé de Turing, où il se bat au corps à corps avec l'argument venu d'une femme, Ada Lovelace. Les thématiques du genre et de l'esprit se croisent en effet dans la mémoire culturelle antique : le mot «esprit» a une longue histoire dans la culture judéo-chrétienne, comme il se trouve être féminin en hébreu (ruah, dans la langue de la Bible hébraïque), neutre en grec (pneuma, dans la langue du Nouveau Testament), puis est devenu masculin au latin (spiritus), et donc dans la théologie chrétienne occidentale. C'est ce dernier usage qui a prévalu durant des siècles.

Dans le corpus des Ecritures judéo-chrétiennes, l'esprit, féminin puis neutre, représente un élément flexible et ouvert. L'esprit, au sens de spirit en anglais, est donc par excellence un lieu de confrontation des genres ou de passage entre les genres. Sans qu'il ne soit besoin de faire ici appel à la biographie de

130 Bush, « As We May Think», 1945, p. 9 : «Consider a future device for individual use, which is a sort of mechanized private file and library. It needs a name, and, to coin one at random, 'memex' will do. A memex is a device in which an individual stores all his books, records, and communications, and which is mechanized so that it may be consulted with exceeding speed and flexibility. It is an enlarged intimate supplement to his memory ».

131 Bush, «As We May Think», 1945, p. 12 : « Presumably man's spirit should be elevated if he can better review his shady past and analyse more completely and objectively his present problems. He has built a civilization so complex that he needs to mechanize his records $[\ldots]$ by overtaxing his limited memory ». 
Turing, faite de souffrance et d'oppression sociale ${ }^{132}$, il est intéressant d'observer la place du genre dans la célèbre expérience mimétique qu'il propose. Il décrit ainsi d'entrée d'article le « jeu de l'imitation » : « Il est joué par trois personnes, un homme (A), une femme (B), et quelqu'un qui interroge (C) qui peut être d'un sexe ou l'autre ${ }^{133}$. Plus loin, il proposera de partir du point de vue masculin : «la meilleure stratégie est d'essayer de fournir des réponses que donnerait spontanément un homme. [...] On pourrait entre autres insister sur le fait que l'équipe des ingénieurs devrait être constituée de personnes toutes du même sexe, mais cela ne serait pas réellement satisfaisant, car il est sans doute possible de tirer un individu complet d'une seule cellule de la peau d'un homme $»^{134}$.

Dans le contexte des années cinquante, un tel point de vue, en milieu académique ou ailleurs, n'est pas surprenant. Néanmoins, on le notera d'autant plus que la bataille argumentative principale de Turing est conduite contre l'objection d'une femme, Ada Lovelace. Une lecture complète de l'article de Turing selon le genre reste à faire et serait profitable à ce pan de l'histoire de l'informatique. Dans le contexte immédiat de ce chapitre, gardons en mémoire la flexibilité de genre de l'esprit-spirit, un mot qui appartient à la thématique de l'esprit que nous scrutons, déjà présente dans l'étude de Menabrea, puis s'étoffant chez Lovelace pour être développée chez Turing, mais sans qu'il utilise le registre de spirit, alors que Vannevar Bush l'intègre en finale de sa réflexion. Faut-il durcir l'alternative? Convient-il d'aligner désormais le mind sur le cerveau, quitte à baptiser «mystère » les découvertes encore à faire, dans la perspective de Turing?

Privilégier une approche généalogique par les noms, enracinée dans la perspective derridienne de l'ouvrage Sauf le nom, c'est être attentif tout autant aux noms manquants qu'aux noms présents, selon le questionnement du philosophe : «Que se passe-t-il surtout quand il faut surnommer, re-nommant là où, justement, le nom vient à manquer ? $\gg^{135}$ C'est pourquoi, tout en reconnaissant la perspective de Turing comme bien plus inspirante que celle de Bush dans l'anamnèse historique des réflexions sur l'intelligence artificielle, il me semble

132 Voir Bernhardt, Turing's vision, édition Kindle, l. 92 ; Alan Turing, article Wikipédia, $<$ https://fr.wikipedia.org/wiki/Alan_Turing> : «En 1952, un fait divers lié à son homosexualité lui vaut des poursuites judiciaires. Pour éviter la prison, il choisit la castration chimique par prise d'oestrogènes. Turing est retrouvé mort dans la chambre de sa maison à Wilmslow, par empoisonnement au cyanure, le 7 juin 1954. La reine Élisabeth II le reconnaît comme héros de guerre et le gracie à titre posthume en 2013 ».

133 Turing, « Computing Machinery and Intelligence », p. 433.

134 Turing, « Computing Machinery and Intelligence », p. 435-436.

135 Derrida, Sauf le nom, première page du feuillet Prière d'insérer. Cité en page 45 ci-dessus. 
capital de creuser cette absence de spirit, à comprendre sans doute comme un geste nécessaire d'émancipation de Turing face aux modèles anthropologique et philosophique de son temps, qui ne convenaient plus très bien à ce qu'il souhaitait exprimer.

Au binôme mind et spirit, avec leur acolyte de chair, le cerveau, je propose d'ajouter la notion de l'impensé ou non-conscient, le unthought du titre de la récente monographie de Katherine Hayles, Unthought. The Power of the Cognitive Nonconscious ${ }^{136}$. De fait, le terme unthought se trouve médité avec mind et spirit dans un long poème de William Wordsworth, The Prelude (1805), dont un vers est cité sur le tout premier logo de la firme Apple ${ }^{137}$. Je choisis d'amener ici dans la réflexion la dimension poétique, car elle fait partie intégrante de l'accès au savoir. En effet, comme le résumait Paul Ricoeur, la poétique révèle des aspects de l'être au monde « qui ne peuvent pas être dits autrement; quand le monde littéraire est poétique, dans un sens plus large que celui de la poésie ou de la narration, il provoque un changement de scène, défamiliarise, en sorte que l'étrangeté dégage la voie pour une appropriation [du monde] complètement nouvelle ${ }^{138}$.

Un vers du Prelude de Wordsworth se trouve donc cité sur le premier logo d'Apple, accompagnant l'image de Newton méditant sous son pommier: «Newton... a mind for ever voyaging through strange seas of thought, alone »139. En entrée de poème, c'est l'esprit-spirit que Wordsworth évoquait : «My spirit was up, my thoughts were full of hope »; plus loin, c'est le tour de l'impensé, en ces termes : «I was obedient as a lute that waits upon the touches of the wind. Unknown, unthought of, yet I was most rich. I had a world about me, 'twas my own ${ }^{140}$.

A l'aide de l'esprit qui pense, mind et spirit, et de l'impensé, unthought, le poète dessine un espace qui navigue entre l'extérieur et l'intériorité (it was my own), mais dépasse aussi cette répartition, puisqu'il s'agit d'« étranges mers de la pensée » (strange seas of thought), des étendues d'eau qui ne semblent guère vouloir être retenues. La notion de l'impensé, du non-conscient, déjà présente en 1805 dans ce poème, me paraît capitale aujourd'hui pour reconsidérer la

136 N. K. Hayles, Unthought. The Power of the Cognitive Nonconscious, University of Chicago Press, 2017.

137 Voir <https://commons.wikimedia.org/wiki/File:Apple_first_logo.png>.

138 P. Ricoeur, «The Canon between the Text and the Community », in P. Pokorny - J. Roskovec (éd.), Philosophical Hermeneutics and Biblical Exegesis, Tübingen, 2002, p. 7-26 ; ici p. 11.

139 W. Wordsworth, The Prelude, 1805, <http://www.bartleby.com/145/ww289.html >, l. 62-63.

140 Wordsworth, The Prelude, <http://www.bartleby.com/145/ww289.html>, l. 18 et 138-140. 
notion de l'esprit et de la pensée face au raz-de-marée en cours de l'intelligence artificielle.

Le unthought est d'ores et déjà en train de faire son chemin dans le monde des Digital Humanities via une monographie de Katherine Hayles (2017). Elle décrivait de fait déjà cette notion en 2012, en se basant sur cet exemple observé durant la Seconde Guerre mondiale :

Une femme qui travaillait à la réception du code en morse [...] durant la seconde guerre mondiale, racontait qu'après ses intenses expériences làbas, elle entendait du code en morse partout - dans le bruit du trafic, dans le chant des oiseaux et dans les autres sons ambiants - et que son esprit formait automatiquement les mots auxquels les sons étaient censés correspondre. Bien qu'aucune donnée scientifique n'existe sur les modifications que la réception du son pourrait opérer sur le fonctionnement nerveux, nous pourrions raisonnablement déduire qu'elle peut provoquer des changements sur le long terme dans les schémas d'activation du cerveau, comme le suggère cette anecdote ${ }^{141}$.

Cette description très physique et concrète des sensations qui sont expérimentées avant d'être conscientisées est également illustrée dans Unthought. Katherine Hayles commence par s'y référer brièvement à un concept Zen, la «pensée sans penser », puis rattache tout de suite le terme aux neurosciences : “' $U n$ thought' peut aussi se référer aux récentes découvertes dans les neurosciences, confirmant l'existence de processus cognitifs non-conscients, des processus inaccessibles à l'introspection consciente, mais néanmoins essentiels pour le fonctionnement de la conscience $»^{142}$. On la sent très vite quitter le cadre d'une pensée négative mystique exprimée par unthought pour aller vers une définition positive et corporelle : alors que le mot unthought se trouve dans le titre, il n'est employé ensuite qu'à la première page de l'ouvrage, puis seulement dans

141 N. K. Hayles, How we think. Digital Media and Contemporary Technogenesis, University of Chicago Press, 2012, p. 127-128; c'est moi qui souligne en mettant une phrase en italiques : «A woman who worked on Morse code receiving [...] during World War II reported that after her intense experiences there, she heard Morse code everywhere - in traffic noise, bird songs, and other ambient sounds - with her mind automatically forming the words to which the sounds putatively corresponded. Although no scientific data exist on the changes sound receiving made in neural functioning, we may reasonably infer that it brought about long-lasting changes in brain activation patterns, as this anecdote suggests ».

142 Hayles, Unthought, p. 1: «'Unthought' may also be taken to refer to recent discoveries in neuroscience confirming the existence of nonconscious cognitive processes inaccessible to conscious introspection but nevertheless essential for consciousness to function ». 
le lexique où il est commenté ainsi : « see nonconscious cognition(s) » ${ }^{143}$. C'est bien donc bien le vocabulaire des « cognitions non-conscientes » qui l'emporte dans l'ouvrage, où l'on note également une forte présence de brain et une présence assez importante de mind. L'esprit au sens de spirit n'est présent que via une citation et dans deux expressions ${ }^{144}$, mais absolument pas comme concept présenté comme tel.

Si les registres de l'approche Zen de l'unthought et de l'esprit-spirit n'ont donc finalement guère de place chez Hayles, on se réjouira en revanche de l'articulation qu'elle met en place entre l'esprit-mind, le cerveau et les cognitions non-conscientes. Un tel dispositif permet d'évoquer la flexibilité de la frontière entre individus et collectivité : les cognitions non-conscientes évoquent « leurs capacités de mutations fluides et de transformations » ${ }^{145}$. C'est dans la flexibilité de cette frontière que se rencontrent les considérations autour de l'esprit-mind et du cerveau biologique, soulignées ainsi par Isabelle Stengers en référence au biologiste Scott Gilbert :

Les faits mènent certains biologistes contemporains à associer les vivants non plus à un mode de composition organismique, avec sa distinction entre individu et milieu, mais à un mode de composition multi-spécifique : « La nature pourrait sélectionner les liens plutôt que des individus ou des génomes. Ce que nous avons l'habitude de considérer comme un 'individu' pourrait être un groupe multi-spécifique soumis à la sélection $»^{146}$.

Il est à relever toutefois que Katherine Hayles, pas plus que Turing, n'articule l'esprit-spirit à sa perspective, alors que la poétique de Wordsworth tient ensemble dans le même parcours méditatif mind, spirit et unthought. J'aimerais ici revendiquer une place propre pour la poétique dans sa capacité à se tenir au croisement du non-conscient, de l'impensé, et de la matérialité. Dans cette perspective, il est très intéressant de constater qu'on trouve une mise en récit, puis une réflexion, sur le phénomène de l'unthought dès les années trente chez l'écrivaine Nathalie Sarraute, née en 1900 en Russie à Ivanovo-Voznessensk, et décédée à Paris en 1999. Son parcours de vie est marqué par une lutte avec une identité doublement minoritaire, femme et juive. Empêchée d'exercer son mé-

143 Hayles, Unthought, p. 249.

144 Hayles, Unthought, p. 188 et 206.

145 Hayles, Unthought, p. 11.

146 Stengers, Civiliser la modernité, édition Kindle, 1. 3143-3150. Elle cite S. F. Gilbert et al., «Symbiosis as a Source of Selectable Epignetic Variation: Taking the Heat for the Big Guy », Philosophical Transactions of the Royal Society B 365 (2010), p. 671-678; ici p. 673. 
tier d'avocate dans les années d'avant-guerre, elle se tourne alors vers l'écriture et publie Tropismes en $1939^{147}$ où des récits rendent compte de la manière dont nous sommes affectés par l'impensé, le unthought, par les mouvements inconscients de notre cerveau, par nos émotions. Pour ce faire, elle emprunte pour le titre de son texte un terme de la biologie végétale, tropisme signifiant une « réaction surprenante des organes d'une plante (racines, tiges, feuilles, fleurs, etc.) à une anisotropie du biotope $»^{148}$. En 1956 , dans L'Ere du soupçon, elle commente ainsi le phénomène qu'elle avait mis en récit dans Tropismes:

Ce sont des mouvements indéfinissables, qui glissent très rapidement aux limites de notre conscience ; ils sont à l'origine de nos gestes, de nos paroles, des sentiments que nous manifestons, que nous croyons éprouver et qu'il est possible de définir. Ils me paraissaient et me paraissent encore constituer la source secrète de notre existence.

Comme, tandis que nous accomplissons ces mouvements, aucun mot pas même les mots du monologue intérieur - ne les exprime, car ils se développent en nous et s'évanouissent avec une rapidité extrême, sans que nous percevions clairement ce qu'ils sont, produisant en nous des sensations souvent très intenses, mais brèves, il n'était possible de les communiquer au lecteur que par des images qui en donnent des équivalents et lui fassent éprouver des sensations analogues. Il fallait aussi décomposer ces mouvements et les faire se déployer dans la conscience du lecteur à la manière d'un film au ralenti. Le temps n'était plus la vie réelle, mais celui d'un présent démesurément agrandi ${ }^{149}$.

Il est fondamental de noter que c'est d'abord dans la sphère littéraire et poétique que surgit la perception et la mise en récit de l'impensé ou du nonconscient, que nous sommes aujourd'hui en train d'articuler à la frange de l'anthropologie, la biologie et la philosophie. Ces aspects ne devraient pas être séparés dans notre manière d'aborder ces nouvelles conceptions de l'humain, de l'individu et de la collectivité. L'esprit-mind est à l'œuvre lorsque nous sommes capables de mettre en lien les dimensions poétique et physique du cerveau pour nous représenter la réalité à nous-mêmes. L'esprit-spirit, par-delà sa connotation théologique, appartient à la sphère poétique et véhicule avec lui une longue tradition de notre capacité à exprimer l'impensé, comme le

147 N. Sarraute, Tropismes, Denoël, 1939.

148 Voir Tropisme, <https://fr.wikipedia.org/wiki/Tropisme>.

149 N. Sarraute, L'Ere du soupçon, Gallimard, 1956, p. 59. 
montre ce passage de Paul de Tarse : «De même aussi l'Esprit nous aide dans notre faiblesse, car nous ne savons pas ce qu'il nous convient de demander dans nos prières. Mais l'Esprit lui-même intercède par des soupirs inexprimables » $(\mathrm{Rm} 8,26)$. L'articulation entre les « soupirs inexprimables » - littéralement non parlés (alalètoï) - de l'esprit-spirit et l'impensé de l'esprit-mind conduisent à situer ce que perçoit notre cerveau au sein d'un cadre culturel. C'est ce même inexprimable que Sarraute décrivait dans le passage cité ci-dessus. Ici se trouve exactement la place requise de la poétique, la théologie pouvant s'entendre comme la poétique portant sur le divin.

On doit ici une grande reconnaissance à Nathalie Sarraute d'avoir si tôt perçu nos impensés, rassemblés autour d'un terme de biologie végétale qui souligne précisément nos sensations non conscientes. Dans le chapitre 3, nous reviendrons à elle autour de la notion du for intérieur qu'elle décline dans son œuvre et que je choisis comme point fondamental pour penser la résistance des sujets et $\mathrm{du}$ «je » des auteurs et lecteurs dans la culture digitale ${ }^{150}$. Pour l'heure, je relèverai, en conclusion de cette deuxième partie du chapitre 2 , que l'esprit-mind qui devient conscient de sa dette au cerveau reste en contact avec sa part spirituelle, aussi longtemps qu'il produit des expressions poétiques, en quête de l'impensé.

\subsection{Le mot de la fin}

Il s'est révélé fructueux, à la suite de la suggestion de Doueihi, de relire Turing. Nous l'avons fait au travers de la thématique spécifique de l'esprit, déclinée chez Turing comme mind entrelacé avec le cerveau. Cette thématique le conduit à mener un face-à-face soutenu avec la figure d'Ada Lovelace autour de son « objection », qu'il ne va toutefois pas relire dans le texte même de Lovelace. Celle-ci se montre bien plus proche du point de vue de Turing qu'il ne l'a considéré, approfondissant la pensée de Menabrea et se montrant consciente des limitations du savoir actuel, soumis sans cesse à la vérification pragmatique de la connaissance. Ces deux figures, féminine et masculine mais très distancées des attentes imposées à leur genre à leur époque, ont été minorisées socialement à ce titre et sont emblématiques pour penser la généalogie des DH. Le « jeu de l'imitation » de Turing démarre de manière implicite sur une mise en scène des genres, avec un élément masculin, un féminin et un non déterminé : dans la généalogie du tournant culturel que nous vivons, ce point de départ est appelé à prendre toute sa place, au côté de la flexibilité grandissante de la frontière entre individu et collectivité, relevée par Stengers. 
En remontant par-delà Lovelace au texte de Menabrea, nous avons également vérifié l'importance de la thématique de l'esprit, présente dès le début de ce texte, jusqu'à l'impensé de l'article de Turing, l'esprit-spirit et le nonconscient, une notion mise à l'honneur dans Unthought de Hayles. Repartant du poème de Wordsworth, lié à l'histoire de l'informatique depuis le premier logo d'Apple, nous avons ensuite trouvé dans l'écriture et l'analyse de Nathalie Sarraute la clé d'articulation des diverses nuances de l'esprit et de l'impensé : la poétique. Cet aspect permet d'honorer ce qu'il y a d'intraduisible dans le nom, comme l'exprimait Jacques Derrida cité en ouverture de chapitre : «Qu'est-ce qui fait du nom propre une sorte de surnom, de pseudonyme ou de cryptonyme à la fois singulier et singulièrement intraduisible ? »151

Cet intraduisible et l'appel au poétique qu'il génère pourraient bien être la machinery lovée au cœur de l'article de Turing. En effet, on constate avec surprise que l'expression utilisée dans le titre, computing machinery, n'est jamais réutilisée dans le corps de l'article. On en reste avec la machinery sans la machine, le terme ayant bien la connotation de ruse qui se trouve en français dans « machinerie » ou «machination ». La résistance du savoir millénaire des humanités se tient dans cet intraduisible, dans la nécessité, dont on n'est jamais dédouané, de s'attacher aux noms et aux mots, et de les visiter en profondeur, entre les langues.

C'est ce qui se révèle tant dans le nom des « humanités numériques ou digitales », que dans celui des digitale Geisteswissenschaften ou des Ruah Digitalit. L'expression francophone ramène en dernier lieu au corps, à ce «moule d'humanité » de la Renaissance, miroir de chair du moule d'imprimerie. Son étude permet de comprendre à quel point la culture digitale provoque le corps et signale son retour au premier plan, par-delà son succédané du corps de papier des écritures, et accompagné du retour du pluriel «humanités », en français. Quant aux expressions allemande et hébraïque, elles lient la thématique de l'esprit à ces humanités digitalisées. L'attention portée à l'article de Turing, et en amont à ceux de Menabrea et Lovelace, ont permis là aussi de faire résonner la présence de ce terme dans l'appropriation que ces deux langues ont faite de l'expression digital humanities. En hommage à Sauf le nom de Jacques Derrida, ce chapitre espère avoir montré combien le regard des humanités, avec sa mémoire des significations et des langages poétiques, occupe une place indispensable dans le déchiffrement des enjeux de la culture digitale.

151 J. Derrida, Sauf le nom, Galilée, 1993. Ce passage se trouve à la première page du feuillet Prière d'insérer qui se trouve glissé au début du volume. 